\title{
Estructura urbana y cambio en el uso del suelo en Bogotá entre 2007 y 2017
}

\author{
Estrutura urbana e mudanças no uso da terra em Bogotá \\ entre 2007 e 2017 \\ Urban structure and land-use change in Bogotá between \\ 2007 and 2017
}

David Felipe Holguín Lozano [a] [D], Juan Camilo Peña Urbina [b] [D]

Andres Dominguez [C] [D,

\begin{abstract}
[a] Universidad de los Andes, Grupo de estudios en sostenibilidad urbana y regional (SUR), Departamento de Ingeniería Civil y Ambiental, Bogotá, Colombia.

[b] Departamento Administrativo Nacional de estadística, Bogotá, Colombia.

[c] Observatorio de Dinámicas Urbano Regionales, Secretaría Distrital de Planeación, Bogotá, Colombia.
\end{abstract}

Cómo citar: Holguin, D., Peña, J., \& Dominguez, A. (2021). Estructura urbana y cambio en el uso del suelo para Bogotá entre 2007 y 2017. urbe. Revista Brasileira de Gestão Urbana, v.13, e20200192. https://doi.org/10.1590/2175-

3369.013.e20200192

\section{Resumen}

El principal objetivo de este documento es estimar un modelo de selección discreta y otro de distribución truncada que permitan identificar los principales determinantes que explican los cambios en el uso del suelo en Bogotá entre 2007 y 2017. Los resultados muestran que las distancias a la malla vial principal, a las troncales de Transmilenio, los centros comerciales y los subcentros de empleo, se encuentran relacionadas con la probabilidad de cambio de uso. Por otro lado, se encontró que las áreas de actividad residencial definidas en el Plan de Ordenamiento Territorial, Decreto 190 de 2004, tienen, en algunos casos, efectos contrarios a los esperados. Los análisis y resultados de este documento son insumos fundamentales para diseñar un sistema normativo que incorpore los intereses de los agentes de una economía, y se pueda tener en cuenta la potencial existencia de conflictos en los usos del suelo y la expansión de la mancha urbana.

Palabras clave: Economía urbana. Uso del suelo. Planeamiento urbano.

DFHL es economista, asistente de investigación, candidato a magister en Economía, e-mail: david.holguin@correo.usa.edu.co JCPU es economista, especialista en Economía urbana y regional, e-mail: juancamilopenaurbina@gmail.com AD es economista, doctor en Economía Aplicada, e-mail: jandresdominguezm@gmail.com 


\section{Resumo}

O principal objetivo deste documento é estimar um modelo de seleção discreta e um modelo de distribuição truncado para identificar os principais determinantes que explicam as mudanças no uso da terra em Bogotá entre 2007 e 2017. Os resultados mostram que as distâncias da malha viária, para os baús Transmilenio, os shopping centers e os subcentros de emprego, estão relacionados à probabilidade de mudança de uso. Por outro lado, verificou-se que as áreas de atividade residencial definidas no Plano de Uso da Terra, Decreto 190 de 2004, têm, em alguns casos, efeitos contrários aos esperados. As análises e os resultados deste documento são insumos fundamentais para projetar um sistema regulatório que incorpore os interesses dos agentes de uma economia, e a possível existência de conflitos no uso da terra e a expansão da mancha urbana podem ser levadas em consideração.

Palavras-chave: Economia urbana. Uso da terra. Planejamento urbano.

\section{Abstract}

The objective of this document is to estimate a discrete selection model and a censored distribution model that specifies to identify the main determinants that explain the changes in land use in Bogotá between 2007 and 2017. The main results point out that distances to the main road, to Transmilenio trunks, shopping centers and employment sub-centers, are related to the probability of change. On the other hand, it is found that areas of residential activity defined in the 2004 Land Use Plan have, in some cases, effects contrary to those expected. The analyzes and results of this document are fundamental inputs to design a regulatory system that incorporates the interests of the agents of an economy, the potential existence of conflicts in land uses and the expansion of the urban slick can be taken into account.

Keywords: Urban economics. Land use. Urban planning.

\section{Introducción}

La estructura espacial de un área urbana está explicada por la distribución de la actividad económica y la actividad residencial, esta estructura puede ser monocéntrica, policéntrica o dispersa. Es posible que la estructura urbana cambie en al menos tres direcciones: incrementos en los niveles de densidad, cambios de uso dentro de estas áreas de actividad económica o la expansión de las concentraciones de actividad económica y de su área de influencia. Esta dinámica puede traducirse en problemas de movilidad vehicular si la infraestructura de transporte no cuenta con la capacidad suficiente.

En una estructura urbana, el Distrito Central de Negocios (DCN) se define como el área con la mayor concentración de actividad económica, mientras que los Subcentros son concentraciones de actividad económica de menor jerarquía separados del DCN. En consecuencia, las presiones que se generan debido las decisiones de localización en la estructura urbana se deben reflejar en los usos del suelo. Por tanto, la hipótesis de trabajo en este documento es que la estructura urbana subyacente explica los cambios de uso del suelo que han ocurrido en el área urbana de Bogotá.

Según cifras del DANE, Bogotá genera la cuarta parte del PIB del país y, en términos de población, registró 6.763.325 habitantes en el Censo de 2005, y 7.181.469 en el Censo de 2018. Este incremento de la población se reflejó en el territorio con incrementos en la densificación de áreas urbanizadas y en la urbanización de áreas de desarrollo. La literatura especializada señala que las áreas en donde se concentra la actividad económica generan condiciones para que las empresas se beneficien de rendimientos crecientes a escala, de la reducción de costos de transporte y de la movilidad de factores productivos (Krugman, 1991).

Además, el incremento en la demanda de suelo para uso residencial y la demanda de suelo por parte de las empresas, generan presiones dentro de la estructura urbana. En consecuencia, en este documento se usan 
métodos cuantitativos para identificar los determinantes que explican cambios en los usos del suelo en el área urbana de Bogotá a nivel de manzana entre 2007 y 2017. Los determinantes son variables que interfieren en la decisión de localización de los agentes: maximizar beneficios, en el caso de las empresas; o maximizar niveles de utilidad, en el caso de los hogares. Específicamente, se estimó un modelo logístico multinomial y un modelo de regresión truncada con el fin de cuantificar la relación de las variables en las decisiones de localización que influyen los cambios de uso del suelo.

Es importante mencionar que el presente documento se enmarca en una discusión actual sobre el modelo de ocupación que definirá el plan de ordenamiento territorial de Bogotá. ${ }^{1}$ Entre las declaraciones que han tomado relevancia en la opinión pública se encuentra la mezcla de usos en zonas de alta densidad para activar dinámicas urbanas y el nivel de acceso que tiene la población a la actividad económica de la ciudad, así como estimular mezclas de usos en zonas con baja densidad con el objetivo de revitalizar sectores urbanos estratégicos. Esto porque la transición de las actividades económicas que se localizan en los primeros pisos, con actividad residencial en los pisos superiores, podría generar dinámicas integradoras. Por tanto, la mezcla de usos permitidos y la implementación de mitigación de impactos se encuentra en un debate que aún no está cerrado.

Además de la presente introducción el documento tiene un marco teórico de referencia, aspectos normativos y revisión de literatura sobre usos del suelo. La sección tres presenta los cambios en los usos del suelo entre 2007 y 2017. En la cuarta sección se encuentra la metodología de los modelos empleados y, en la quinta sección, los resultados. La última sección se ocupa de las conclusiones.

\section{Marco Teórico}

En la literatura sobre modelos de localización se menciona el de von Thünen (1826). Este modelo explica la localización de actividades agrícolas teniendo en cuenta los costos de transporte. En la literatura más reciente, Alonso (1964), Kristof (1970) y Mills (1967) retoman el modelo de von Thünen (1826) y desarrollan modelos sobre la distribución de los usos del suelo en entornos urbanos. Los supuestos principales parten de una estructura monocéntrica, en donde el costo de transporte está en función de la distancia al DCN.

Estos modelos incorporan el concepto de bid rent, en donde los agentes tienen incentivos para localizarse cerca del mercado en donde se efectúan las transacciones, con el fin de disminuir costes de transporte. De hecho, en los entornos urbanos, los productores relacionados con actividades de comercio y servicios tienen incentivos para localizarse en el DCN. Ellos compiten por el uso del suelo, contra agentes que realizan actividades asociadas a la industria o al uso residencial.

Estos conceptos teóricos permiten tener una idea de la distribución de los usos en el espacio urbano, las densidades de empleo, precios del suelo y demás. Sin embargo, al realizar un contraste empírico con la distribución actual del suelo en las ciudades existen diferencias importantes. Por ejemplo, Odland (1978) concluyó que una estructura monocéntrica no describe satisfactoriamente las ciudades modernas. En esa línea, diferentes autores como Fujita \& Ogawa (1982), McDonald \& Prather (1994) o Muñiz et al. (2008) incorporan estructuras policéntricas. En este escenario, cada trabajador que es contratado por una firma debe asumir los costos de desplazamiento entre su residencia al lugar de trabajo (Fujita \& Krugman, 2004).

Adicionalmente, Lucas \& Rossi-Hansberg (2002) — en adelante Modelo LRH — desarrollan un modelo de ciudad circular en donde agregan el concepto de uso mixto para describir la localización de los agentes en una economía urbana y el uso que se hace del suelo. Esto debido a que existen lugares en las ciudades donde los

\footnotetext{
1 El vigente Plan de Ordenamiento Territorial (POT) de Bogotá se adoptó por medio del Decreto 190 de 2004 y se han realizado dos procesos de actualización que no han tenido éxito, uno en 2013 y otro en 2019. Actualmente se avanza en el proceso de actualización y se espera tener un nuevo POT en el año 2022 (SDP, 2021).
} 
agentes económicos tienen una disposición a pagar por el uso del suelo similar tanto para usos económicos como para usos residenciales, lo que se materializa un uso mixto del suelo. La Figura 1 muestra el uso del suelo en diferentes anillos concéntricos que conforman la estructura urbana.

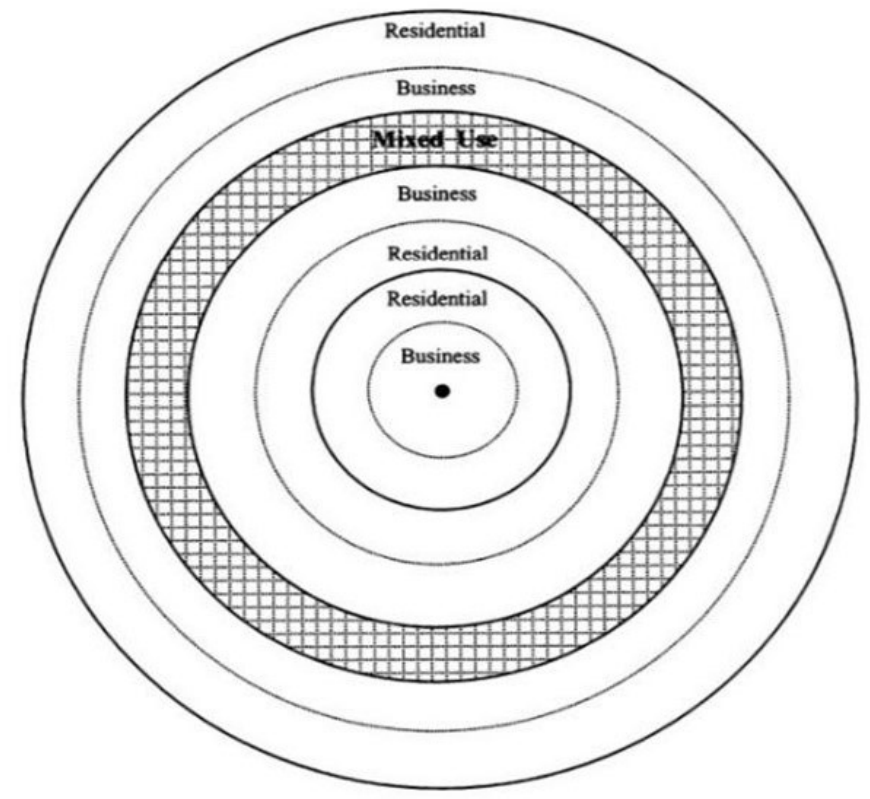

Figura 1 - Modelo de estructura urbana LRH. Fuente: Lucas \& Rossi-Hansberg (2002).

Adicionalmente, la localización de los agentes y los usos que le dan al suelo pueden modificarse mediante incentivos, estos incentivos pueden generarse a través de la construcción de equipamientos urbanos, vías, etc. por parte de la administración pública. Jaramillo (2009) argumenta que:

Una primera forma de incidencia del Estado en el mercado de tierras en la ciudad se desprende de su acción como agente constructor... Estas operaciones, en la medida que alteran y transforman la estructura urbana introducen cambios en los precios de los terrenos, y no solamente en los lotes desarrollados por el Estado, sino en aquellos que se relacionan espacialmente con ellos (p. 329).

Dependiendo del tipo de inversión estatal y la distancia a dicha inversión la disposición a pagar y los usos que se le dan al suelo cambian; por ejemplo, una inversión en mejoramiento vial se traduce en una disminución generalizada en los costos de transporte, lo que genera incentivos para que los agentes decidan vivir en localizaciones más alejadas del DCN. Este efecto no solamente incide en las periferias de la ciudad, sino también en aquellos lugares donde la disposición a pagar por el uso residencial disminuyó ya que se generan incentivos de localización en los agentes que desarrollan otros usos de actividad económica.

La provisión de infraestructuras dotacionales también incrementa el precio de los inmuebles que se localizan en cercanías de estas inversiones, estos incrementos en los precios pueden ocasionar que se supere la disposición a pagar que se tenía por la renta del inmueble para cierto uso, lo que puede ocasionar un cambio parcial o total del uso que se le da al inmueble.

Además de intervenir mediante la construcción de bienes públicos locales y el mejoramiento vial, la administración pública también interviene en los usos del suelo mediante la disposición de normas: reglamentación de alturas y usos permitidos. Dichas disposiciones condicionan la estructura urbana ocasionando que en ciertos sectores de la ciudad la renta que se percibe por la actividad económica sea diferente a lo que se percibiría sin estas restricciones; un ejemplo de ello es cuando se limitan los lugares donde 
se puede ejercer el uso comercial. En este documento se estudia si dichas medidas tienen el efecto esperado sobre los usos del suelo, ya que no en todas las ocasiones los agentes acatan las disposiciones normativas.

La intervención de la administración pública en la estructura urbana tiene incidencia directa en el surgimiento o consolidación de los DCN, ya que estas zonas de la ciudad se caracterizan por ser altamente densas, tener muy buena accesibilidad, estar cerca de infraestructuras dotacionales, entre otras. A continuación, se mencionan algunos de estos aspectos normativos para la ciudad de Bogotá.

\section{Aspectos normativos}

En Colombia, la Ley 388 de 1997 (Congreso de Colombia, 1997) regula el uso del suelo y es el principal soporte legal de los Planes de Ordenamiento Territorial (POT). Un POT es el marco jurídico para la actuación urbanística. En el caso de Bogotá, las decisiones consignadas en el POT — Decreto 190 de 2004 (Alcaldía Mayor de Bogotá, 2004) — se estructuran espacialmente en Unidades de Planeación Zonal (UPZ). A nivel de UPZ se definen:

- Lineamientos de estructura urbana de cada Unidad.

- Regulación de la intensidad y mezcla de usos.

- Condiciones de edificabilidad.

- Lineamientos sobre el manejo de ruido acorde con la política ambientales.

Adicionalmente, se normativizan las áreas de actividad y los tratamientos urbanísticos. En las áreas de actividad se define la posibilidad y las condiciones en las cuales se puede desarrollar un uso. Con respecto a tratamientos, en este documento se estudia el tratamiento de consolidación, el cual regula la transformación de la ciudad construida y se aplica a manzanas en donde las características urbanísticas están consolidadas (en particular se analizará la densificación moderada y consolidación urbanística). Sin embargo, realizar un uso diferente al establecido en la norma suele tener grandes incentivos económicos. Por ello en la metodología cuantitativa se incorporan variables normativas con el fin de identificar si dichas restricciones tienen el efecto esperado en la probabilidad de cambio de uso del suelo.

\section{Revisión de la literatura}

Los procesos de urbanización, particularmente los que se desarrollan de manera acelerada, generan problemas relacionados con la relocalización de actividades económicas, la concentración de la vivienda en zonas periféricas (con problemas de transporte tanto público como privado) y los conflictos entre usos del suelo destinados a la vivienda y a la actividad económica (Peréz \& Polése, 1996).

El estudio empírico de las estructuras urbanas ha sido abordado de manera extensa. Por ejemplo, McDonald \& Prather (1994) detectan la existencia de tres Subcentros de empleo en Chicago. Los autores analizan gradientes de densidad (la tasa a la cual disminuye la densidad de empleo a medida que se incrementa la distancia al DCN). Resultados similares obtiene McMillen (2004) para 62 áreas metropolitanas de EEUU; donde encuentra que, para todas las ciudades, los Subcentros de empleo tienen una relación negativa con la densidad de empleo a medida que la distancia aumenta.

Para Bogotá, Avendaño et al. (2014) identificaron Subcentros basándose en la metodología de Muñiz et al. (2008) y Muñiz \& García-López (2010). Los autores estimaron el efecto del DCN y los Subcentros sobre los precios del suelo. Los resultados permiten categorizar a Bogotá como una estructura urbana policéntrica. Para el caso de los Subcentros, encuentran que los sectores cercanos tienen un precio que disminuye conforme aumenta la distancia. En términos de usos, se espera que las unidades espaciales localizadas cerca al DCN y Subcentros tengan baja participación de uso residencial y a medida que la distancia aumenta, esta participación aumente. 
Por otro lado, Koster \& Rouwendal (2013) realizan una aproximación empírica en Holanda según el Modelo LRH. Dentro de sus hallazgos está la existencia de áreas mixtas, en donde las empresas y los hogares tienen disponibilidad a pagar similares. Esto supone que las empresas deciden localizarse en áreas residenciales y debido a que ofrecen más renta logran expulsar a algunos hogares. En consecuencia, estas áreas empiezan a consolidarse con usos mixtos del suelo.

Para encontrar las características que determinan los cambios de uso en el tiempo, se han utilizado modelos logísticos multinomiales. Por ejemplo, el Departamento Administrativo de Planeación (2016), utiliza estos modelos para pronosticar la atractividad que tienen las áreas de intervención estratégica de Medellín en cuestiones de localización de vivienda, con el fin de obtener una estimación de la transición espacial del recaudo por aprovechamientos urbanísticos. Este modelo de elección sobre localización explica las decisiones que toman los hogares y determinan las características que inciden en la ubicación de las viviendas.

La metodología que se usa en el presente documento es similar a la utilizada por Xu et al. (2013) para Pinggu, en Beijing. Los autores realizan un análisis de cambio de uso del suelo cultivable a otros usos. Por ejemplo, a usos como asentamientos, huertas, carreteras o pastizales en el periodo de 1993-2008. Mediante el ejercicio descriptivo de tabulación cruzada se logra identificar el cambio absoluto en cada uso y para encontrar los determinantes de los cambios se estima un modelo logístico multinomial. El presente documento aborda un contexto urbano, cuyo objetivo es explicar los usos del suelo a nivel de manzana entre 2007 y 2017 teniendo en cuenta las restricciones normativas sobre el cambio de uso urbano.

Por último, para Bogotá, Domínguez (2017) estudia el cambio de uso en manzanas residenciales. Sin embargo, existen, al menos, tres diferencias con respecto a la metodología propuesta en el presente documento: (i) la inclusión de la regulación existente de los usos del suelo, (ii) el periodo de análisis, y (iii) la metodología que utiliza Domínguez (2017) se plantea desde los modelos probabilísticos binomiales.

La importancia de estos análisis radica en que, como lo señalan Eliasson et al. (2020), a través de las inversiones de infraestructura de transporte (líneas de metro) los formuladores de política generan cambios en los valores del suelo y, a través de ello, el cambio en los usos del suelo. En esta línea, Zheng et al. (2016) investiga el impacto que han tenido las estaciones de metro en las amenidades del vecindario, puesto que la construcción de una estación incrementa el número y la diversidad de restaurantes en la zona. Por su parte, Bowes \& Ihlandfeldt (2001) muestran como las estaciones de trenes no solamente incrementan el valor de las propiedades, sino que también atraen actividades económicas de comercio al por menor, lo que significa cambios en los usos del suelo.

\section{Cambios en los usos del suelo}

El presente documento plantea que la estructura urbana tiene la capacidad de explicar los potenciales cambios en los usos del suelo en las zonas de influencia tanto del DCN como de los Subcentros de empleo. Los Subcentros de actividad económica se obtienen de Avendaño et al. (2014) con información del año 2005 (Figura 2). La utilización de información espacial rezagada en el tiempo permite explicar cambios de uso del suelo controlando potenciales problemas de endogeneidad o doble causalidad. 


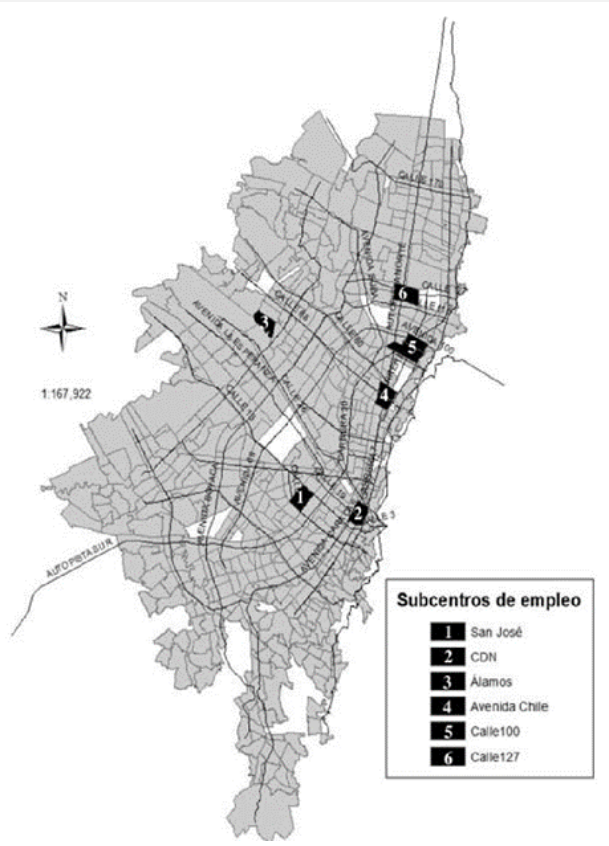

Figura 2 - Subcentros de empleo, Bogotá, 2005. Fuente: Avendaño et al. (2014).

La Figura 3 muestra el porcentaje de manzanas por categoría de uso para 2007 y 2017 en un área de influencia del DCN y los Subcentros de la Figura 2. Los usos del suelo se identificaron con información de la Infraestructura de Datos Espaciales para el Distrito Capital (IDECA), de tal manera que se asignó una categoría de uso a cada manzana (residencial, mixto, y comercio y oficinas) y se evalúa la participación con respecto a la distancia con respecto al DCN o Subcentro más cercano.
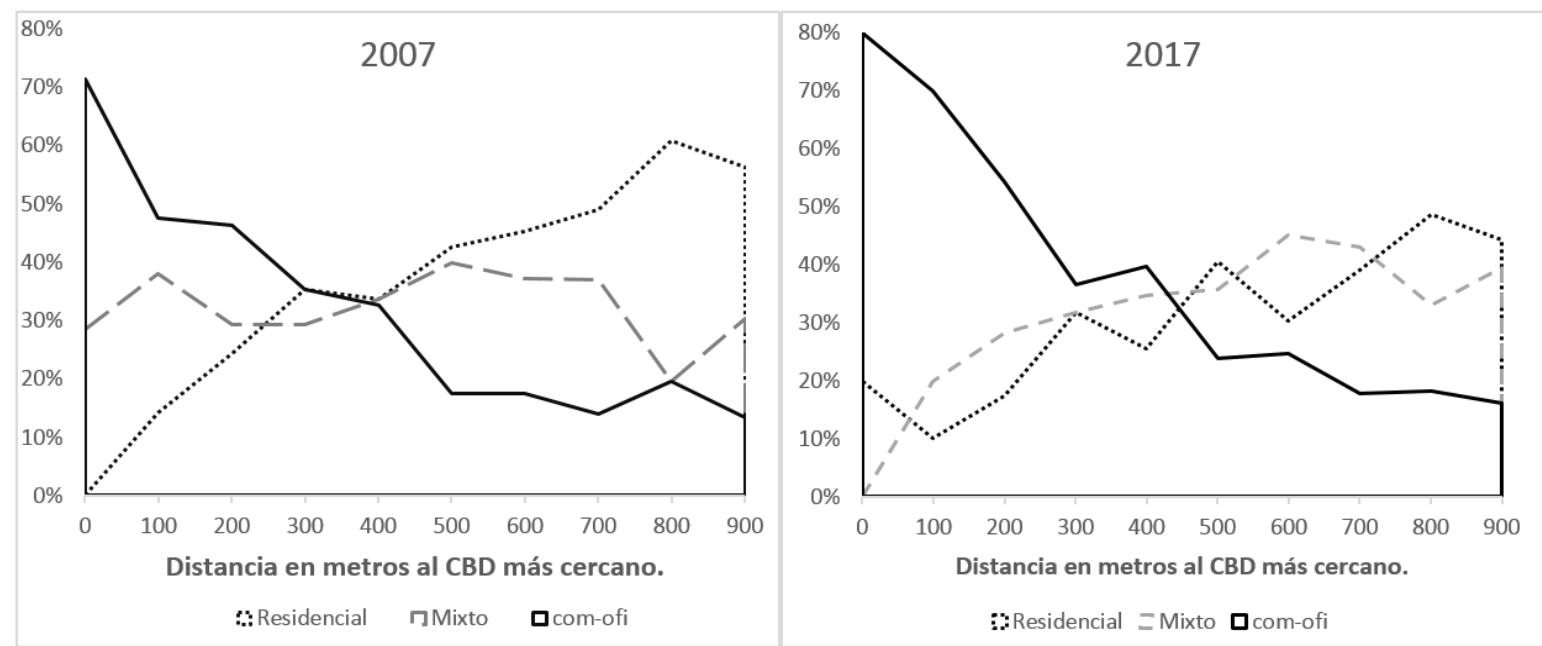

Figura 3 - Porcentaje de manzanas por clasificación del suelo según la distancia al centro o subcentro más cercano. Fuente:

Cálculos propios con información de IDECA para los años 2007 y 2017 (www.ideca.gov.co)..

En 2007 la línea más oscura muestra que el porcentaje de manzanas con uso de comercio y oficinas se reduce a medida que la distancia se incrementa (desde 70\% a 10\% cuando la distancia es de 900 metros). Mientras que el porcentaje de manzanas con uso residencial se incrementa de $0 \%$ a 55\% (línea punteada). La línea fragmentada muestra cómo el porcentaje de manzanas con uso mixto se mantienen por debajo del 30\%. Para 2017, aunque el porcentaje de uso comercial y oficinas disminuye (desde $80 \%$ hasta menos del $20 \%$ ), el porcentaje de las manzanas con uso residencial se incrementa levemente del $10 \%$ al $40 \%$ y las manzanas con 
uso mixto en algunas localizaciones superan el porcentaje de manzanas con uso de comercio y servicios y residencial. Es decir que las manzanas con uso residencial disminuyen dando paso a usos mixtos.

La información de IDECA clasifica 98 categorías de uso. Con dicha información se conformaron 13 grupos de usos: comercio grande, comercio pequeño, dotacional, otros, industria grande, industria pequeña, oficinas, restauranteshoteles, transporte-alimentos y 4 tipos de uso residencial (la Tabla A1 del Anexo el ejercicio de agrupación).

La clasificación de usos del suelo se encuentra a nivel de lote, y debido a que cada manzana puede tener diferentes lotes con diferentes usos, se calculó el porcentaje de área construida por manzana y por uso:

$$
p_{i}=\frac{\sum_{n=1}^{m} s_{i}}{a}
$$

En la ecuación (1), $p_{i}$ representa el porcentaje de área construida de una manzana con el uso i; $m$ el número de lotes de la manzana; $s_{i}$ los metros construidos del uso i en los lotes de una manzana; y $a$ el área total construida de la manzana. Entonces, para cada manzana se identificó el uso con mayor porcentaje de área construida y ese uso se considera como uso predominante de la manzana. La información estadística evidencia que el $75 \%$ de área construida en la ciudad tiene uso residencial, por tanto, las manzanas residenciales se separan en 4 opciones diferenciadas por el porcentaje de predominancia.

En la Tabla 1 se reporta la matriz de transición que contiene la cantidad de manzanas por categoría de uso predominante para 2007 y 2017. En la diagonal principal se encuentran las manzanas que mantuvieron su uso en la década de estudio, mientras que en el resto de la matriz se encuentra el número de manzanas que cambiaron de un uso a otro. El ejercicio se realizó con 38,548 manzanas.

Tabla 1 - Matriz de transición de usos predominantes 2007-2017

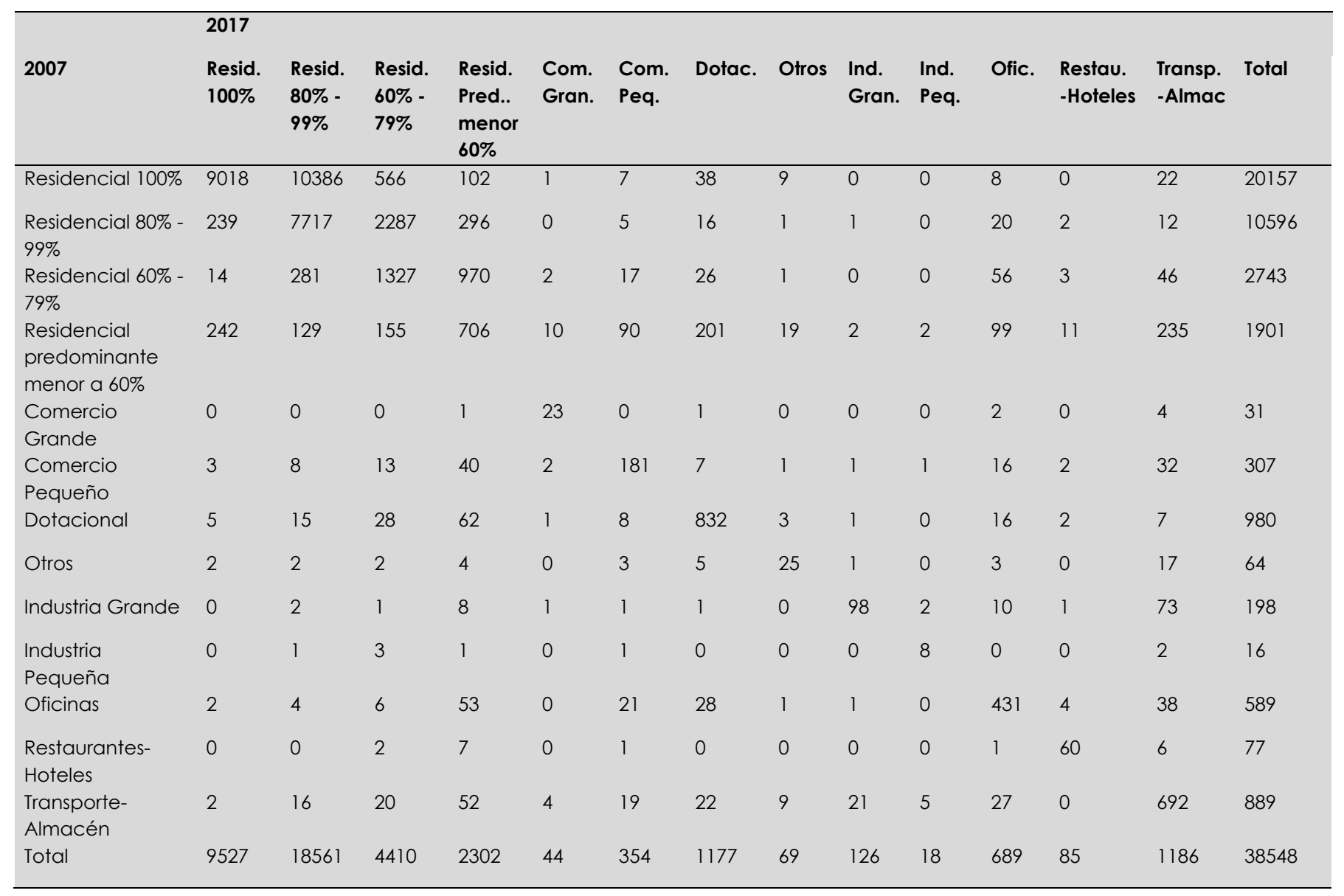

Fuente: Cálculos propios con información de IDECA para los años 2007 y 2017 (www.ideca.gov.co).

urbe. Revista Brasileira de Gestão Urbana, 2021, 13, e20200192 
Los datos de la matriz de transición indican que el 48.3\% de las manzanas cambió de categoría. Dentro de ese porcentaje, el 78.7\% de manzanas la predominancia de uso residencial disminuyó dando paso a otros usos, es decir que se generó mayor mezcla. La mayor cantidad de casos de cambio de uso se presenta en las manzanas con alta participación de uso residencial y que de manera gradual el uso económico fue ganando participación.

\section{Metodología}

\section{Elección discreta}

En términos metodológicos, la modelación econométrica permite obtener valores entre 0 y 1 que se obtienen de una función de probabilidad para cada una de las entidades espaciales, en este caso manzanas. Los modelos Logit, a todos los valores estimados entre 0.5 y 1 , por defecto les asigna el valor 1; mientras que a los valores que se encuentran por debajo de 0.5 les asigna el valor 0 , y así se obtiene la dicotomía entre el cambio del uso del suelo o no de cada manzana incluida en el análisis.

La probabilidad de cambio de uso se describe como: $\operatorname{Pr}\left(Y_{i}=1 / X_{i}\right)=F\left(X_{i} \beta\right)$. Este tipo de modelos permiten calcular los efectos marginales de cada una de las variables explicativas por el método de máxima verosimilitud. La probabilidad se define en la ecuación (2):

$$
\pi_{j}=\frac{e^{\left(\beta_{j 0}-\beta_{j 1} x_{1 i}+\cdots+\beta_{j k} x_{k i}\right)}}{\sum_{h=1}^{k} e^{\left(\beta_{j 0}-\beta_{j 1} x_{1 i}+\cdots+\beta_{j k} x_{k i}\right)}}
$$

Donde $i$ representa las unidades espaciales (manzanas); $k$ las variables independientes del modelo; $j$ es la cantidad de categorías del modelo logístico; y $\beta$ es el coeficiente para estimar asociado a cada variable. Los efectos marginales se calculan de acuerdo a la ecuación (3):

$$
\frac{\partial \operatorname{Pr}\left(Y_{i}=1 / X_{i}\right)}{\partial X_{i k}}=\frac{\partial F\left(X_{i}^{\prime} \beta\right)}{\partial X_{i k}}=f(X \beta) \beta_{k}
$$

Donde $F$ es la función de distribución y $f$ es la función de densidad. Si el efecto marginal es igual a 0 indicaría que la variable independiente no tiene relación con la probabilidad de cambio de uso del suelo, mientras que, si el efecto marginal es cercano a 1 o -1, la variable tiene una relación significativa con el cambio del uso del suelo.

En términos económicos, los modelos de elección discreta (Logit y Probit) estiman la probabilidad de una alternativa dentro de un conjunto de posibilidades. La alternativa con mayor probabilidad es aquella que maximiza la utilidad de un agente (McFadden, 1974). Posteriormente McFadden (1977) estudia la localización de las familias teniendo en cuenta los atributos de cada alternativa de ubicación posible mediante un modelo de elección discreta multinomial. El supuesto principal es que la ubicación seleccionada maximiza la utilidad.

Dado que la variable dependiente tiene más de dos categorías, la literatura especializada señala que es posible plantear la estimación de modelos binomiales o modelos multinomiales. Para identificar cuál es la mejor especificación, Hausman y McFadden (1984) proponen una prueba estadística con el fin de establecer si se cumple la propiedad de independencia de alternativas irrelevantes, si se cumple la mejor especificación es la del modelo multinomial. En el caso específico del análisis que se lleva a cabo en el presente documento, la hipótesis nula reveló que se debe estimar un modelo multinomial. 
Por último, el modelo multinomial se puede estimar con la especificación Logit o Probit, con el fin de identificar el modelo de probabilidad que mejor se ajusta a los datos, se realizaron dos pruebas de criterios de información (la Tabla A2 del Anexo reporta el AIC propuesto por Akaike (1974) y el BIC propuesto por Schwarz (1978)). Los estadísticos sugieren que el modelo con mejor ajuste es el modelo logístico multinomial, Logit.

\section{Variable dependiente}

La Tabla 1 muestra las tres categorías de la variable dependiente en el modelo logístico multinomial. La categoría es 0 si el uso predominante de la manzana era 100\% residencial en 2007 y no se presentó cambio de uso para 2017; categoría 1 si una manzana que era 100\% residencial en 2007 disminuyó a un rango entre 80\% y 99\% en 2017; y categoría 2 si la predominancia disminuyó a un rango entre $60 \%$ y 79\%. De tal manera que la variable dependiente captura de manera progresiva los cambios de uso residencial a otros usos (ver Figura 4).

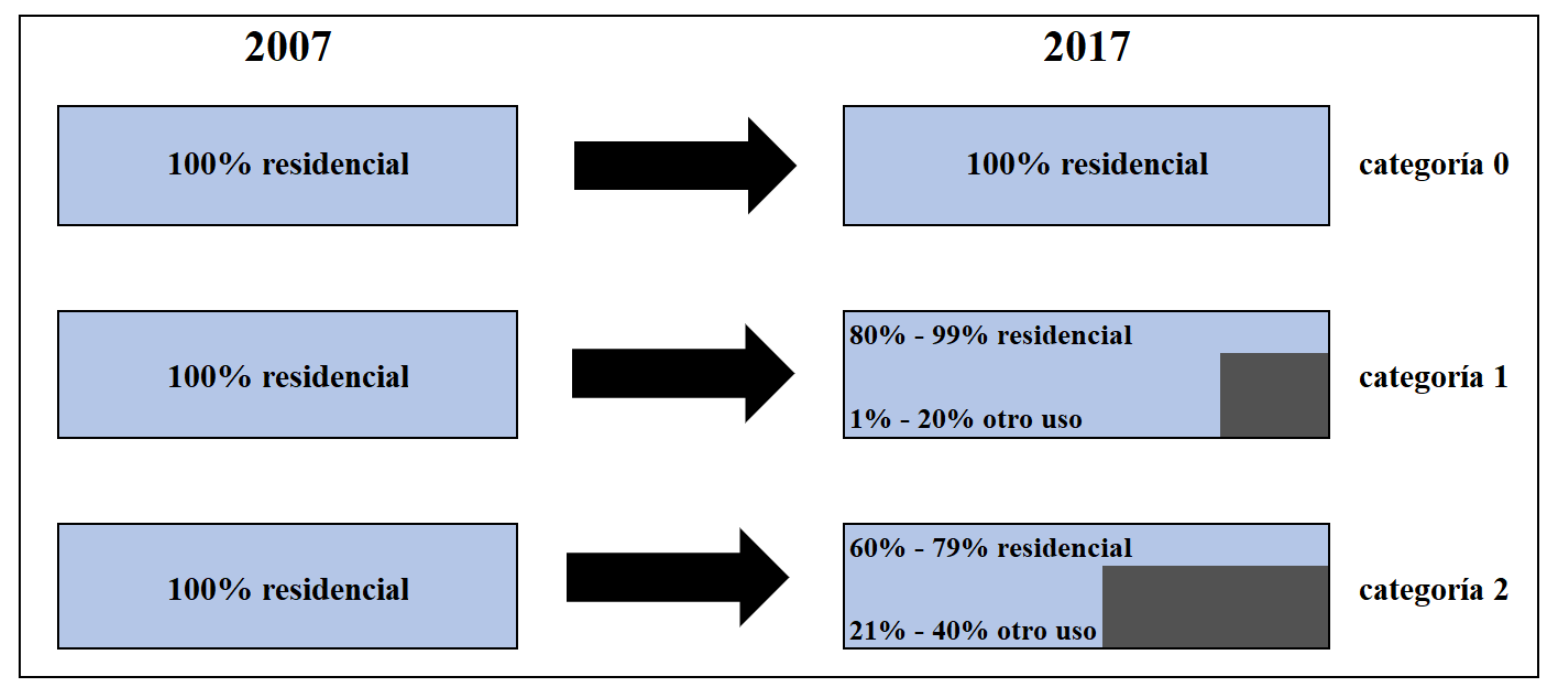

Figura 4 - Categorías de la variable dependiente. Fuente: Elaboración propia.

La Figura 5 muestra las manzanas que en el 2007 tenían uso 100\% residencial, y la escala de azules indica el porcentaje de metros cuadrados residenciales construidos que tenían las manzanas en 2017. El Panel A muestra el caso de la localidad de Usaquén, en esta zona de la ciudad se observa que los porcentajes residenciales disminuyeron en la mayoría de las manzanas que en 2007 eran cien por ciento residenciales. El Panel B muestra las localidades Usme y San Cristóbal, se evidencia un comportamiento inverso al de la localidad de Usaquén, ya que, en esta zona de la ciudad, si bien se evidencia que algunas manzanas presentan disminución en los porcentajes residenciales, la mayoría de las manzanas mantuvo el uso residencial exclusivo. 


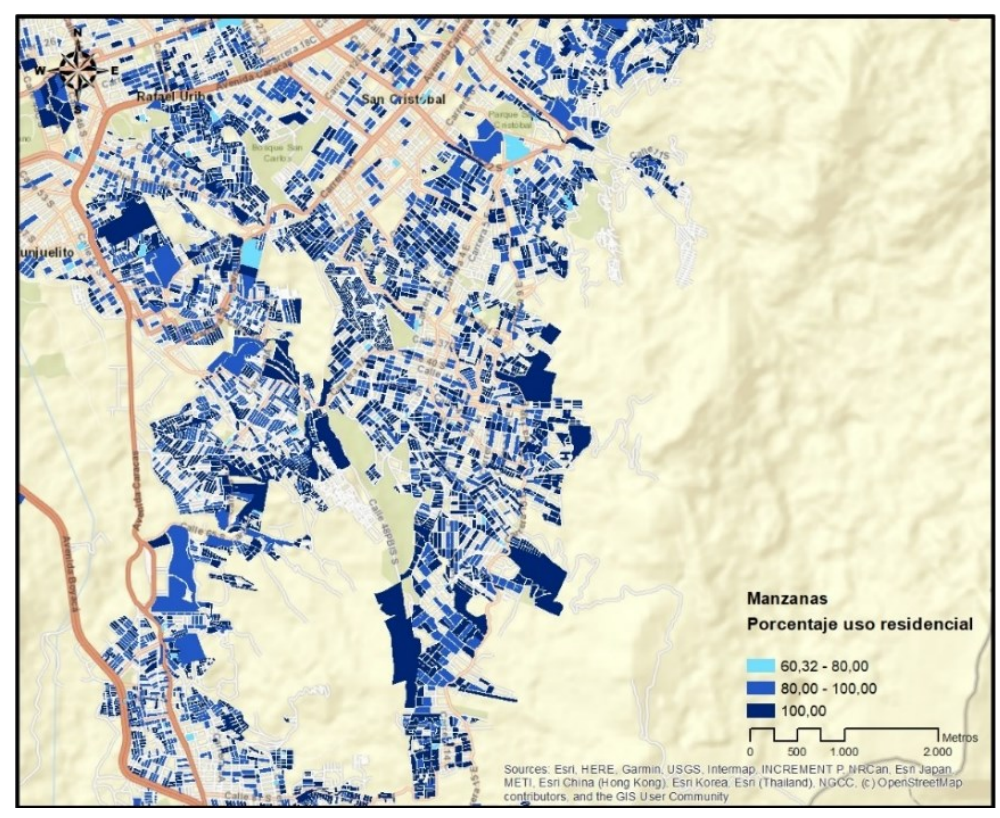

(a)

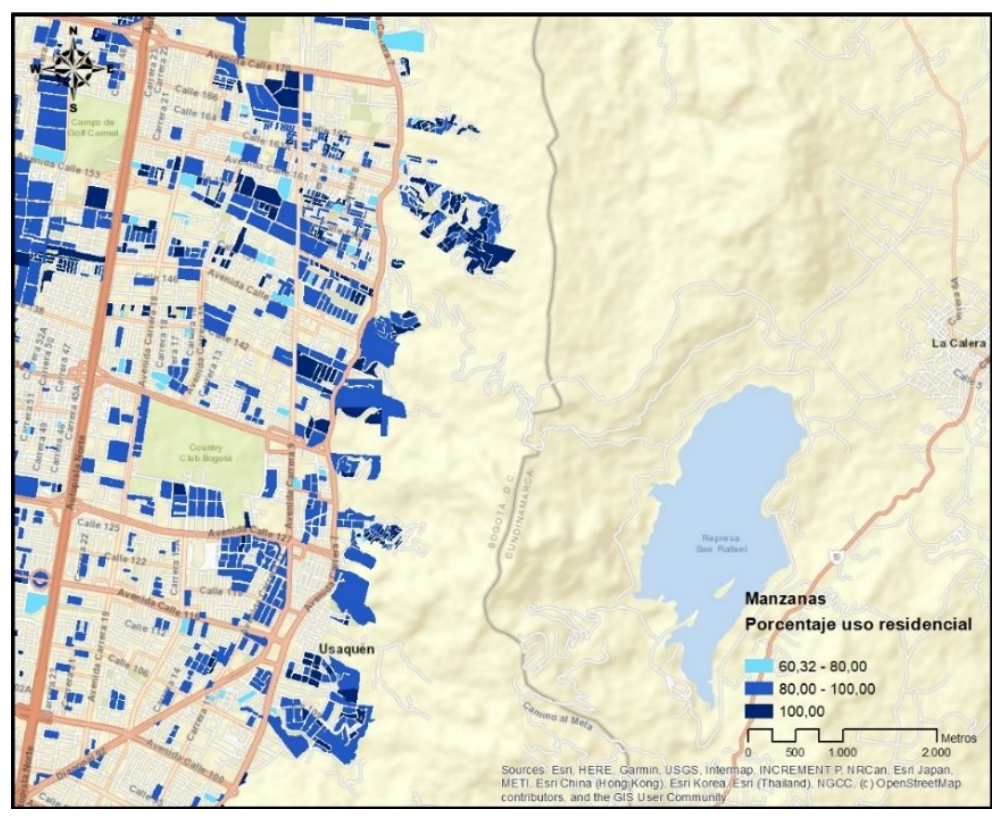

(b)

Figura 5 - Porcentaje residencial de 2017 para manzanas 100\% residenciales en 2007: (a) Suroriente de la ciudad, Localidades Usme y San Cristóbal (b) Nororiente de la ciudad, localidad de Usaquén. Fuente: Elaboración propia.

\section{Modelo truncado}

Con el fin de estimar un modelo asumiendo la variable dependiente continua, se estimó un modelo de regresión Tobit. Esta metodología permite truncar el valor de la variable dependiente en alguna parte de su distribución. En este caso, la variable dependiente queda definida como el porcentaje de uso residencial de la manzana para el año 2017. Entonces, el máximo valor que puede asumir la variable es de $100 \%$. Por tanto, se censura la distribución después de este valor hacia la derecha de la distribución. 
Tanto las variables explicativas como las observaciones de este modelo fueron las mismas incorporadas en el modelo multinomial. Formalmente se especifica la ecuación (4):

$$
y_{i}=\left\{\begin{array}{cl}
y_{i}^{*}=\beta_{0 i}+X_{i k} \beta_{k 1}+u_{i}, & \forall y *_{i}<100 \\
100, & \forall y *_{i} \geq 100
\end{array}\right.
$$

Donde $y_{i}{ }^{*}$ es una variable latente que sigue la misma distribución de la variable dependiente en la parte no truncada; $X_{i k}$ la matriz de variables con i observaciones y k variables; $\beta_{k 1}$ el vector de coeficientes; y $u_{i}$ el termino error. La función de densidad debe definirse por partes como se describe en la ecuación (5):

$$
f\left(y_{i} \mid X_{i}\right)=\left\{\begin{array}{cr}
f\left(y_{i}{ }^{*} \mid X_{i}\right), & \text { si } D_{i}=1 \\
\operatorname{Pr}\left(y_{i}=100 \mid X_{i}\right), & \text { si } D_{i}=0
\end{array}\right.
$$

Donde $D_{i}$ asume el valor de 1 si la variable latente es menor a 100 y el valor de 0 en el caso contrario. El supuesto de normalidad de los errores permite que las distribuciones se comporten como se describe en las ecuaciones (6) y (7):

$$
\begin{gathered}
f\left(y_{i}{ }^{*} \mid X_{i}\right)=\varphi\left(\frac{x_{i}^{\prime} \beta}{\sigma}\right) \\
\operatorname{Pr}\left(y_{i}=100 \mid X_{i}\right)=\operatorname{Pr}\left(y_{i}{ }^{*} \geq 0\right)=\theta\left(\frac{-x_{i}^{\prime} \beta}{\sigma}\right)=1-\theta\left(\frac{x_{i}^{\prime} \beta}{\sigma}\right)
\end{gathered}
$$

Una vez determinada la función de densidad es posible realizar el proceso de optimización de la función de log-verosimilitud que queda definida en la ecuación (8):

$$
\log L\left(\beta, \sigma^{2}\right)=\sum_{i=1}^{n} D_{i} \log \varphi\left(\frac{x^{\prime} \beta}{\sigma}\right)+\left(1-D_{i}\right) \log \left(1-\theta\left(\frac{x_{i}^{\prime} \beta}{\sigma}\right)\right)
$$

Como resultado, los valores de los coeficientes que maximizan la función son los apropiados.

\section{Variables explicativas}

La metodología propuesta se diseñó con el objetivo de incluir variables normativas y variables de localización espacial. Como se mencionó anteriormente, los Subcentros de empleo son determinantes para explicar los gradientes de uso del suelo. Por tanto, se calculan la distancian de las manzanas al Subcentro de empleo más cercano, esta variable se representa en el modelo como lndsc. En el mapa de la Figura 6 se muestran las distancias en desviaciones estándar. El DCN y subcentros son los calculados por Avendaño et al. (2014) (Figura 2). 


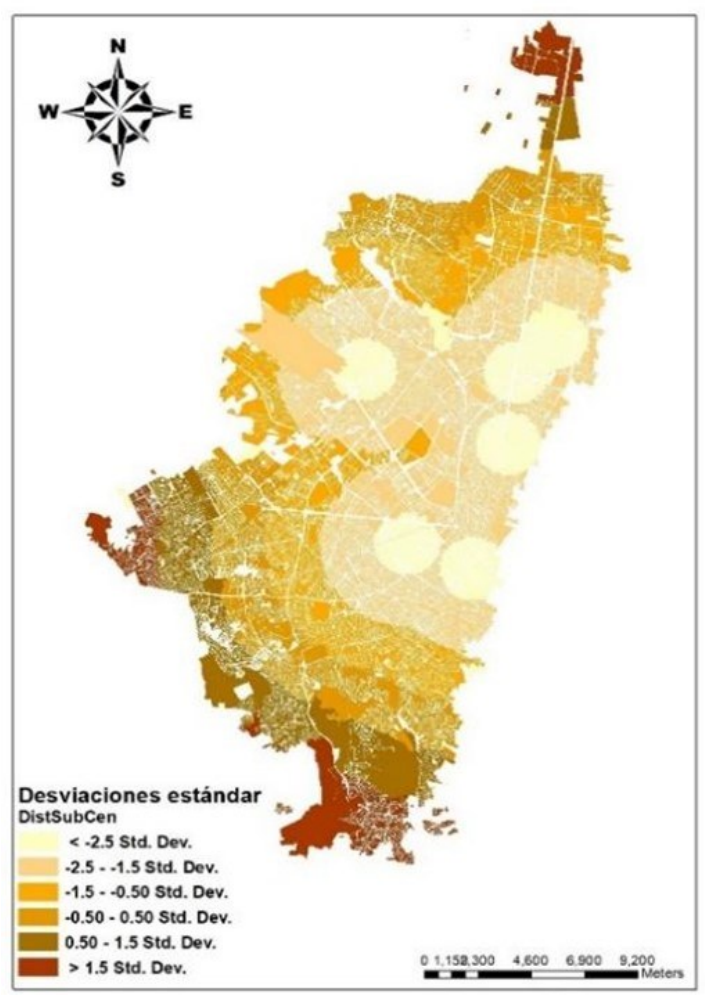

Figura 6 - Distancia de cada manzana al CDN y Subcentros. Fuente: Cálculos propios con información de IDECA para los años 2007 y 2017 (www.ideca.gov.co).

La literatura menciona que las variables asociadas a la movilidad y el acceso a transporte público son elementos fundamentales para explicar el uso efectivo y los cambios en los usos del suelo (Vergel-Tovar, 2016; Rodríguez et al., 2016). Por lo tanto, se calcula la distancia de cada manzana a la malla vial principal de la ciudad y a las estaciones de Transmilenio, estas variables se incorporan en el modelo como: lndmvi para la distancia a malla vial principal y lndtm para las distancias a la estación de Transmilenio más cercana. Los mapas de las Figuras 7 y 8 muestran estas variables. 


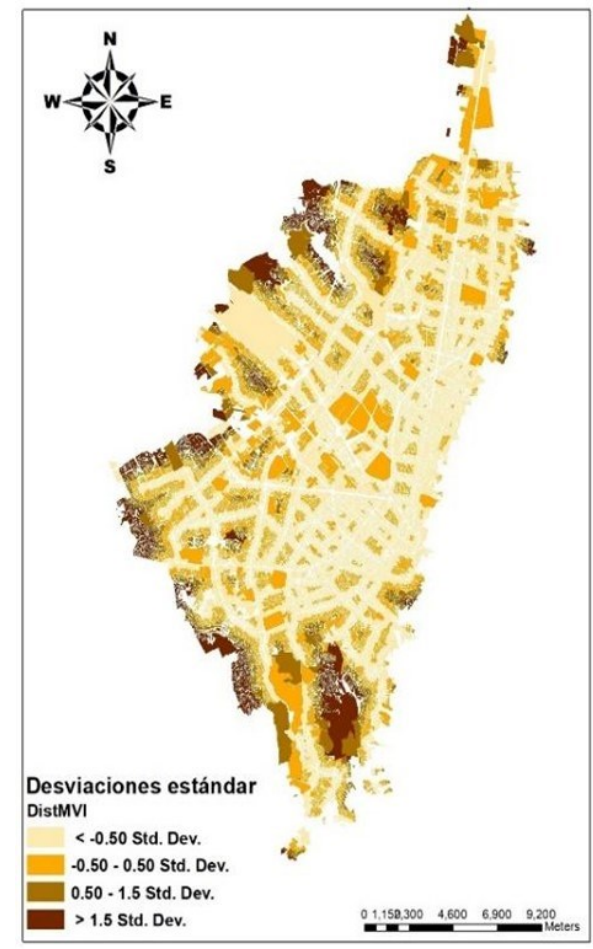

Figura 7 - Distancia de cada manzana a la malla vial principal. Fuente: Cálculos propios con información de IDECA para los años 2007 y 2017 (www.ideca.gov.co).

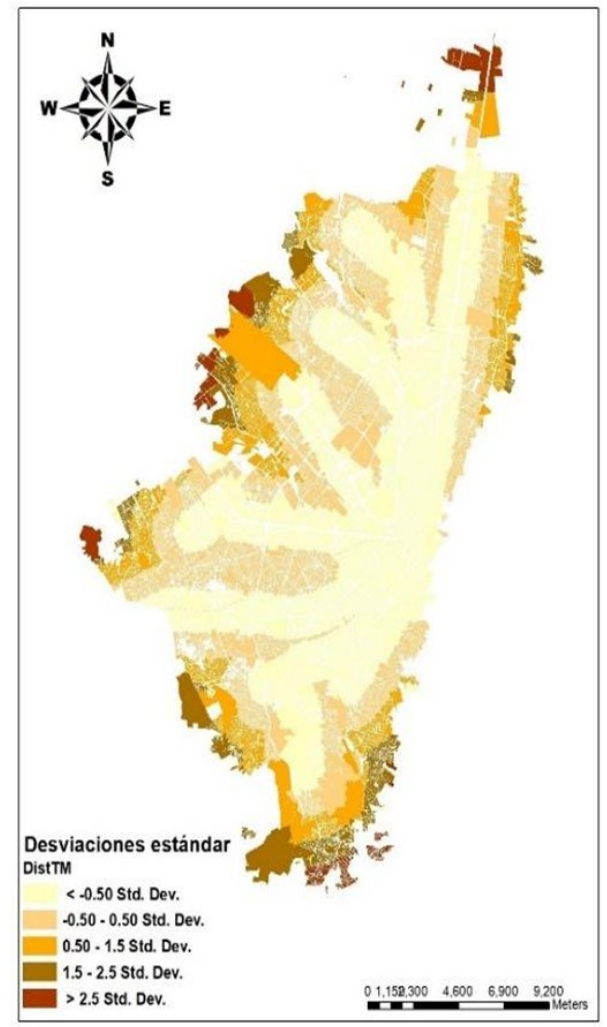

Figura 8 - Distancia de cada manzana a estaciones de Transmilenio. Fuente: Cálculos propios con información de IDECA para los años 2007 y 2017 (www.ideca.gov.co). 
También se incorporó la distancia de las manzanas hasta los centros comerciales más cercanos (lndcc). Existen incentivos en las manzanas cercanas a los centros de comercio a ofrecer bienes o servicios complementarios o sustitutos a los ofrecidos en los centros de comercio, lo que puede explicar los cambios de uso del suelo. Los centros comerciales seleccionados fueron aquellos que se encontraban construidos en el año 2011 (con el fin de evitar problemas de endogeneidad). La Figura 9 representan las distancias de esta variable en desviaciones estándar.

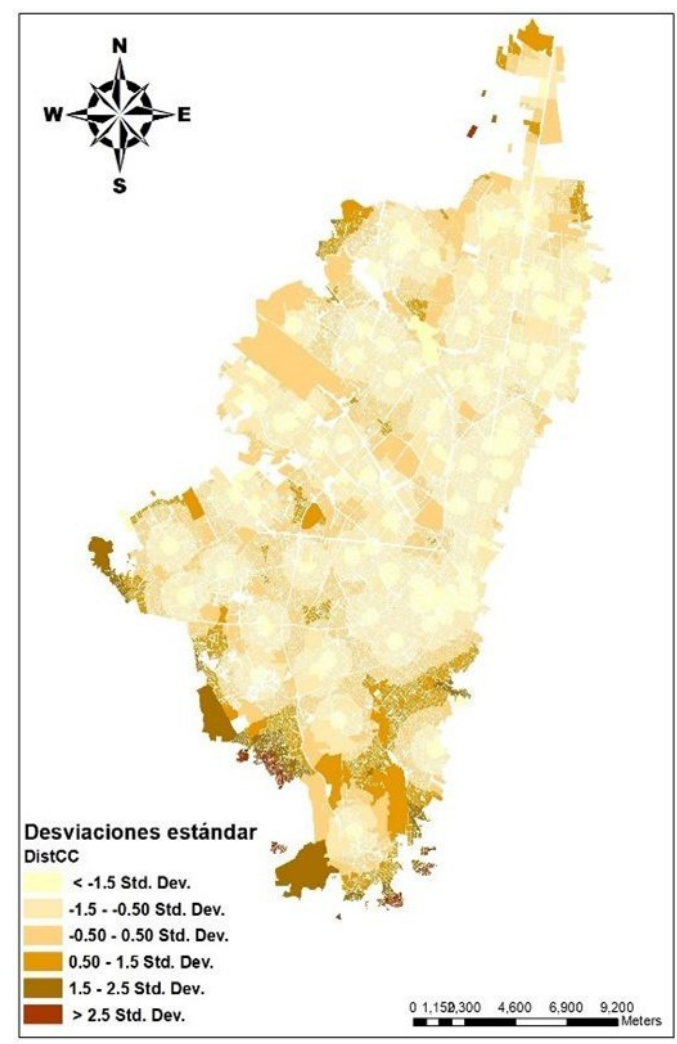

Figura 9 - Distancia de cada manzana a centros comerciales. Fuente: Cálculos propios con información de IDECA para los años 2007 y 2017 (www.ideca.gov.co).

Para incluir los aspectos normativos en las áreas de estudio, los modelos econométricos incorporan dos subtratamientos urbanísticos y áreas de actividad. Los tratamientos urbanísticos fueron establecidos por normas que definen manejos diferenciados según las características físicas de cada zona de la ciudad. Se incorporaron dos subtratamientos: Consolidación con Densificación Moderada, cdm, ${ }^{2}$ y Consolidación Urbanística, curb. ${ }^{3}$ Estas variables binomiales toman el valor de 1 si la manzana está categorizada con el subtratamiento y 0 si en otro caso. La Figura 10 muestra las manzanas con estos dos subtratamientos.

\footnotetext{
2 El subtratamiento de consolidación urbanística (curb) delimita sectores residenciales de la ciudad que cuentan con adecuadas condiciones urbanísticas. En estos sectores la administración desea mantener este patrón urbano.

3 Áreas que por los cambios constructivos de la ciudad han modificado el modelo original y han generado un cambio del patrón urbanístico, en este caso la administración desea consolidar el nuevo patrón urbano.
} 


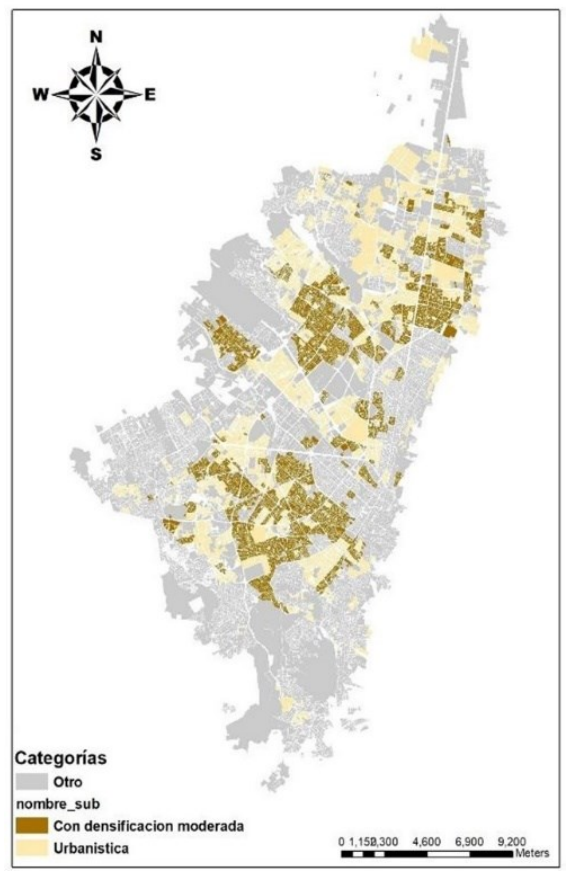

Figura 10 - Tratamientos urbanísticos, Decreto 190 de 2004. Fuente: Cálculos propios con información de IDECA (para los años 2007 y 2017 (www.ideca.gov.co).) y Decreto 190 de 2004.

Por último, las áreas de actividad reflejan los usos asignados a cada zona de la ciudad. En este documento se tiene en cuenta las áreas de actividad residencial, y comercio y servicios. Se incorporan en el modelo como actres y actcys, respectivamente. Estas variables toman el valor de 1 si la manzana se encuentra en el área de actividad y 0 en otro caso. La Figura 11 muestra las manzanas por áreas de actividad.

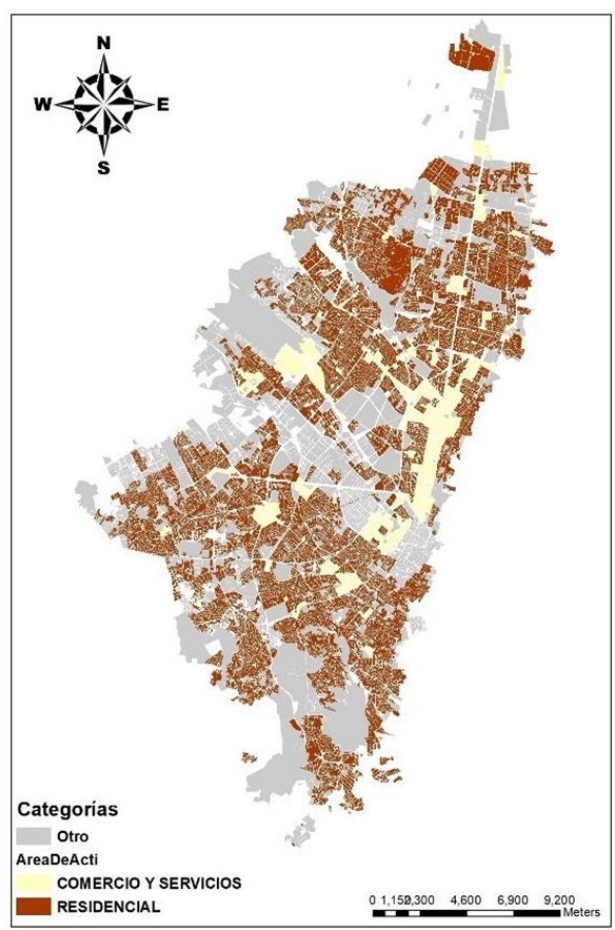

Figura 11 - Áreas de actividad, Decreto 190 de 2004. Fuente: Cálculos propios con información de IDECA y Decreto 190 de 2004. 
Con el fin de identificar si algunas de las variables se encuentran correlacionadas entre sí se reporta una tabla de correlaciones entre las variables explicativas. Esto para detectar potenciales problemas de multicolinealidad en los modelos de regresión (la Tabla A3 del Anexo reporta la matriz de correlaciones).

\section{Estimaciones y resultados}

\section{Modelo multinomial}

Al estimar el modelo multinomial se obtienen las ecuaciones de probabilidad para cada una de las categorías del modelo tal como se describe en las ecuaciones (9), (10) y (11):

$$
\begin{aligned}
& \pi_{0}=\frac{1}{\psi} \\
& \pi_{1}=\frac{e^{\left(\beta_{01}+\beta_{11} l n d c c+\beta_{21} l n d m v i+\beta_{31} l n d s c+\beta_{41} \text { lndtm }+\beta_{51} \text { curb }+\beta_{61} c d m+\beta_{71} \text { actcys }+\beta_{81} \text { actres }+\beta_{91} \text { pointx }+\beta_{10} \text { pointy }\right)}}{\psi} \\
& \pi_{2}=\frac{e^{\left(\beta_{02}+\beta_{12} \text { lndcc }+\beta_{22} \text { lndmvi }+\beta_{32} \text { lndsc }+\beta_{42} \text { lndtm }+\beta_{52} \text { curb }+\beta_{62} c d m+\beta_{72} \text { actcys }+\beta_{82} \text { actres }+\beta_{91} \text { pointx }+\beta_{10} \text { pointy }\right)}}{\psi}
\end{aligned}
$$

Donde $\pi_{i}$ es la probabilidad estimada de cada categoría. $\psi$ representa la suma de todos los numeradores del cociente asociado a cada probabilidad como se especifica en la ecuación (12):

$$
\begin{gathered}
\psi=1+e^{\left(\beta_{01}+\beta_{11} \text { lndcc }+\beta_{21} \text { lndmvi }+\beta_{31} \text { lndsc }+\beta_{41} \text { lndtm }+\beta_{51} c u r b+\beta_{61} c d m+\beta_{71} \text { actcys }+\beta_{81} \text { actres }\right)}+ \\
e^{\left(\beta_{02}+\beta_{12} \text { lndcc }+\beta_{22} \text { lndmvi }+\beta_{32} \text { lndsc }+\beta_{42} \text { lndtm }+\beta_{52} c u r b+\beta_{62} c d m+\beta_{72} \text { actcys }+\beta_{82} \text { actres }\right)}
\end{gathered}
$$

En la Tabla 2 se presentan los efectos marginales de las variables sobre la probabilidad de cambio en la predominancia del uso residencial. Con el fin de controlar la relación espacial entre las manzanas se incorporaron las coordenadas $(x, y)$ de los centroides de las manzanas, pointx y pointy respectivamente.

\section{Modelo Truncado}

La variable dependiente observada en la parte no censurada se describe en la ecuación (13):

$$
\begin{gathered}
\widehat{y}_{l}=\widehat{y}_{l}^{*}=\widehat{\beta}_{0}+\widehat{\beta}_{1} \operatorname{lnd} c c+\widehat{\beta}_{2} \ln d m v i+\widehat{\beta}_{3} \ln d s c+\widehat{\beta}_{4} \ln d t m+\widehat{\beta}_{5} c u r b+\widehat{\beta}_{6} c d m+ \\
\widehat{\beta}_{8} \text { actres }+\widehat{\beta}_{9} \text { point }+\widehat{\beta}_{10} \text { pointy }
\end{gathered}
$$

El valor de la variable en la parte truncada se describe en la ecuación (14):

$$
\begin{gathered}
\widehat{\operatorname{Pr}}\left(y^{*} \geq 100\right)=\theta\left(\hat{\beta}_{0}+\hat{\beta}_{1} \operatorname{lnd} c c+\hat{\beta}_{2} \ln d m v i+\hat{\beta}_{3} \ln d s c+\hat{\beta}_{4} \operatorname{lndtm}+\hat{\beta}_{5} c u r b+\hat{\beta}_{6} c d m+\hat{\beta}_{7} \text { actcys }+\hat{\beta}_{8} \text { actres }+\right. \\
\hat{\beta}_{9} \text { point }+\hat{\beta}_{10} \text { pointy }
\end{gathered}
$$

En la Tabla 2 se encuentran los efectos marginales asociados a las tres categorías del modelo multinomial, y los coeficientes del modelo Tobit. 
Estructura urbana y cambio en el uso del suelo para Bogotá entre 2007 y 2017

Tabla 2 - Efectos marginales modelo multinomial y coeficientes del modelo censurado

\begin{tabular}{|c|c|c|c|c|c|}
\hline \multirow[t]{3}{*}{ Variable explicativa } & \multirow[t]{3}{*}{ Abreviación } & \multicolumn{3}{|c|}{ Modelo logístico multinomial } & \multirow[t]{3}{*}{ Modelo Tobit } \\
\hline & & Categoría 0 & Categoría 1 & Categoría 2 & \\
\hline & & $d y / d x$ & $d y / d x$ & $d y / d x$ & \\
\hline \multirow{4}{*}{$\begin{array}{l}\text { Log - distancia a centro } \\
\text { comercial más cercano. } \\
\text { Distancia a la estación de } \\
\text { Transmilenio más cercana } \\
\text { (logaritmo). }\end{array}$} & \multirow[t]{2}{*}{ Indcc } & $0.040^{* * *}$ & $-0.041^{* * *}$ & 0 & $0.506^{* * *}$ \\
\hline & & $(-0.0069)$ & $(0.0069)$ & $(0.0019)$ & $(0.1270)$ \\
\hline & \multirow[t]{2}{*}{ Indtm } & $0.008^{*}$ & -0.004 & $-0.004^{* * *}$ & $0.368^{* * *}$ \\
\hline & & $(-0.0052)$ & $(0.0052)$ & $(0.0013)$ & (0.0943) \\
\hline \multirow{2}{*}{$\begin{array}{l}\text { Distancia a malla vial } \\
\text { principal más cercana } \\
\text { (logaritmo). }\end{array}$} & \multirow[t]{2}{*}{ Indmvi } & $0.052^{* * *}$ & $-0.043^{* * *}$ & $-0.009 * * *$ & $1.297^{* * *}$ \\
\hline & & $(-0.0045)$ & $(0.0045)$ & $(0.0011)$ & $(0.0827)$ \\
\hline \multirow{2}{*}{$\begin{array}{l}\text { Distancia a subcentros de } \\
\text { empleo (logaritmo). }\end{array}$} & \multirow[t]{2}{*}{ Indsc } & $0.068^{* * *}$ & $-0.067^{* * *}$ & -0.001 & $1.196^{* * *}$ \\
\hline & & $(-0.0093)$ & (0.0092) & (0.0023) & $(0.1637)$ \\
\hline \multirow{2}{*}{$\begin{array}{l}\text { Tratamiento de } \\
\text { consolidación con } \\
\text { densificación moderada. }\end{array}$} & \multirow[t]{2}{*}{ curb } & $0.085^{* * *}$ & $-0.073^{* * *}$ & $-0.012^{* * *}$ & $1.463^{* * *}$ \\
\hline & & $(0.0102)$ & $(0.0101)$ & $(0.0024)$ & $(0.19118)$ \\
\hline \multirow{4}{*}{$\begin{array}{l}\text { Tratamiento de } \\
\text { consolidación urbanística. } \\
\text { Áreas de actividad } \\
\text { residencial. }\end{array}$} & \multirow[t]{2}{*}{$\mathrm{cdm}$} & $-0.110^{* * *}$ & $0.108^{* * *}$ & 0.002 & $-1.058^{* * *}$ \\
\hline & & $(0.01296)$ & (0.0129) & (0.0033) & (0.2352) \\
\hline & \multirow[t]{2}{*}{ actcys } & $-0.112^{* *}$ & 0.033 & $0.079^{* * *}$ & $-6.488^{* * *}$ \\
\hline & & (0.0449) & $(0.0454)$ & $(0.0223)$ & (0.7799) \\
\hline \multirow{2}{*}{$\begin{array}{l}\text { Áreas de actividad } \\
\text { comercio y servicios. }\end{array}$} & \multirow[t]{2}{*}{ actres } & $-0.077^{* * *}$ & $0.089 * * *$ & $-0.012^{* *}$ & -0.179 \\
\hline & & $(0.0151)$ & $(0.0149)$ & $(0.0053)$ & $(0.2855)$ \\
\hline \multirow[t]{2}{*}{ Coordenada X. } & \multirow[t]{2}{*}{ pointx } & $0.000 * * *$ & $0.000^{* * *}$ & 0 & $0.002^{* * *}$ \\
\hline & & (0) & (0) & (0) & $(0.00002)$ \\
\hline \multirow[t]{2}{*}{ Coordenada Y. } & \multirow[t]{2}{*}{ pointy } & $0.000 * * *$ & $0.000^{* * *}$ & $0.000^{* * *}$ & $-0.002^{* * *}$ \\
\hline & & (0) & (0) & (0) & $(0.00001)$ \\
\hline
\end{tabular}

Nota: Errores estándar en paréntesis. ${ }^{* *} p<0.01,{ }^{* *} p<0.05,{ }^{*} p<0.1$. Fuente: Cálculos propios.

Si bien no es posible comparar los resultados de ambos modelos, es importante que los signos de los coeficientes sean consistentes entre ambos modelos: Dado que la variable dependiente del modelo truncado está definida como el porcentaje de uso residencial en 2017 se puede concluir que la dirección de los efectos en ambos modelos es la misma.

Al georreferenciar los resultados del modelo econométrico, se observa que hacía el sur de Bogotá (localidades: Usme, San Cristóbal y Ciudad Bolívar), es más probable que la manzana se encuentre en la categoría 0 (mantenga el uso residencial 100\%), mientras que los sectores de la ciudad que más probabilidad tienen de cambiar el uso residencial a otros usos se encuentran en localidades como Engativá, Suba y Kennedy (Figura 12). 


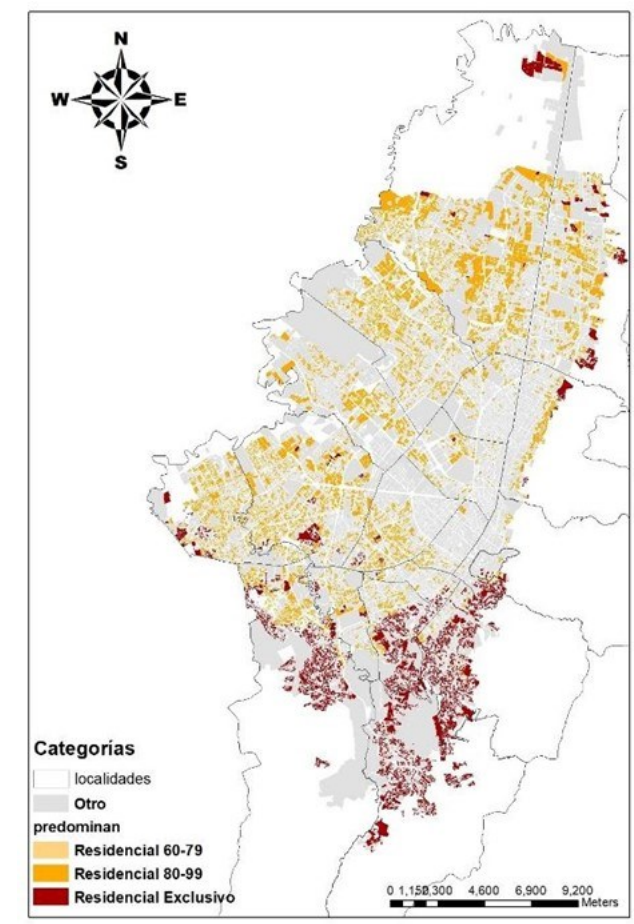

Figura 12 - Probabilidades de cambio de uso de las manzanas. Fuente: Cálculos propios.

Las mayores probabilidades de cambio de uso a la categoría 1, se encuentran en Suba, Engativá, Kennedy, Puente Aranda y Fontibón. También se concluye que no existe una zona determinada en donde el uso predominante residencial $100 \%$ cambie a la categoría 2 . Probablemente la demanda de suelo para uso residencial se está trasladando de las zonas de categoría 1 a la categoría 0 , esto indica que en las zonas donde predomina la categoría 1 se han creado presiones que ocasionan que la disposición a pagar de los agentes no compense los beneficios de realizar otro uso.

\section{Análisis de robustez}

En el año 2007, el 2.29\% de los lotes englobados por construcciones con uso residencial eran de Propiedad Horizontal $^{4}(\mathrm{PH})$. Este tipo de construcciones presentan restricciones en los cambios de uso, porque para ejercer un uso distinto al residencial debe ser aprobado por los copropietarios en asamblea general. En estos casos el uso no está determinado por las circunstancias de la localización, sino por la naturaleza jurídica de las construcciones.

Existen dos tipos de construcciones con uso residencial en propiedad horizontal: los edificios y los conjuntos residenciales. Estos últimos tienen una restricción más fuerte al cambio de uso porque por lo general presentan un número mayor de copropietarios. En esta sección se compara el modelo logístico multinomial presentado en la sección anterior y una estimación de ese modelo que restringe las manzanas englobadas por conjuntos residenciales ( $\sin \mathrm{PH})$.

En la Tabla 3, se muestran los coeficientes para las Categorías 1 y 2 del Modelo sin restricción y del modelo sin PH. Se puede observar que los coeficientes de las variables explicativas tienen el mismo signo en ambos modelos. En términos de magnitud se observa que los coeficientes del logaritmo de la distancia a los centros de empleo lndcc y consolidación urbanística Curb del modelo sin PH son menores a los del modelo original. Por

\footnotetext{
${ }^{4}$ Forma especial de dominio en la que concurren derechos de propiedad exclusiva sobre bienes privados y derechos de copropiedad sobre el terreno y los demás bienes comunes.
} 
otro lado, los coeficientes de las variables explicativas cdm y actcys, en magnitud, son mayores en el modelo sin $\mathrm{PH}$.

Tabla 3 - Análisis de Robustez

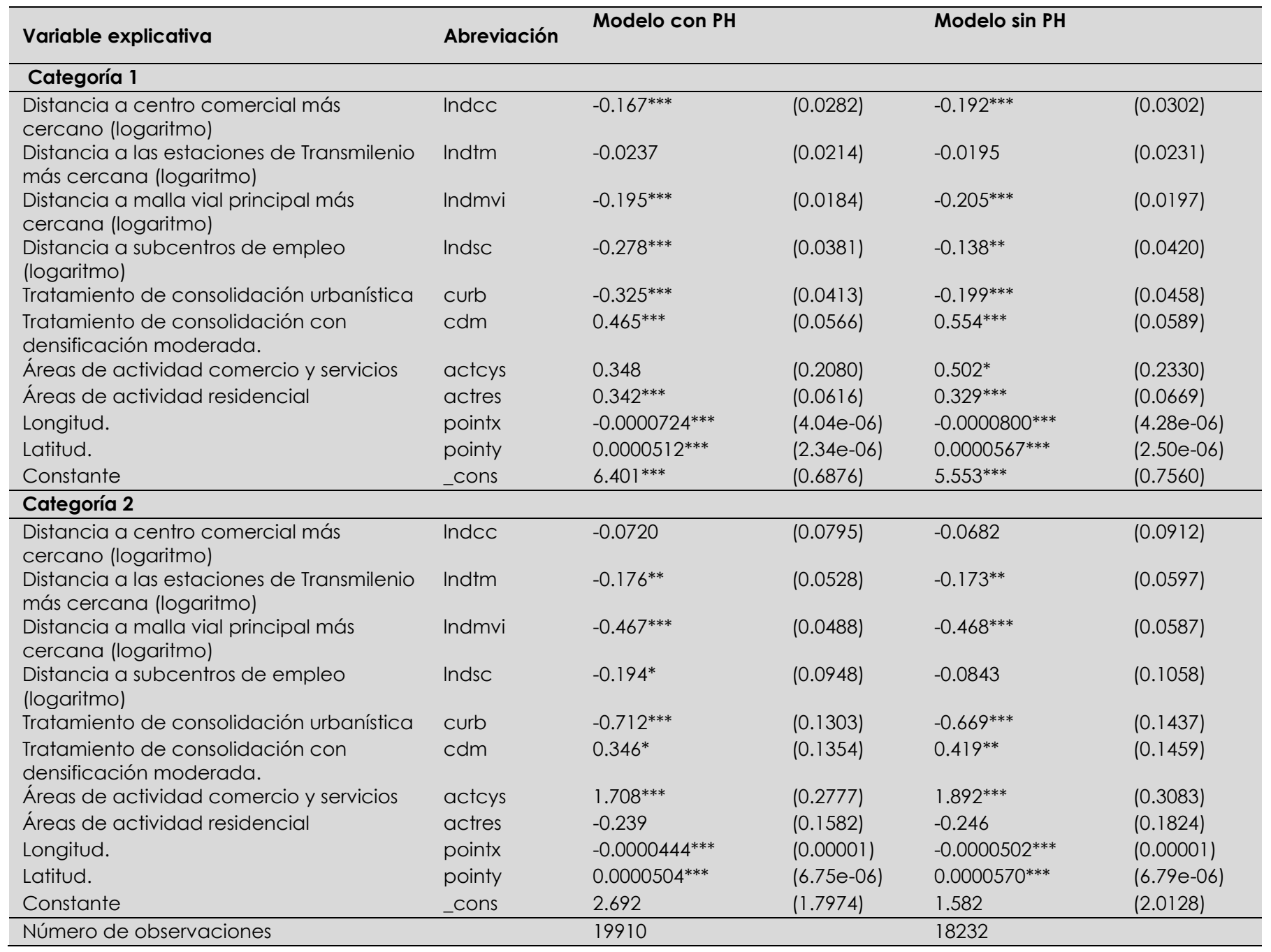

Nota: Errores estándar en paréntesis. ${ }^{* * *} p<0.01,{ }^{* *} p<0.05,{ }^{*} p<0.1$. Fuente: Cálculos propios.

El modelo restringido demuestra que si no se excluyen las manzanas englobadas por conjuntos residenciales se podrían cometer errores de tipo I y tipo II en la significancia individual de los coeficientes. El error tipo II se presenta en la variable actcys de la categoría 1, ya que se asume que no es significativo estadísticamente cuando en realidad lo es. Por otro lado, se comete error tipo I porque lndcc en la categoría 2 para el modelo original es significativa, mientras que para el modelo sin PH no lo es.

\section{Conclusiones}

Los procesos de urbanización, particularmente los que se desarrollan de manera acelerada, generan problemas la relocalización de actividad económica, diferenciación progresiva entre el DCN y centro histórico, la concentración de la vivienda en zonas periféricas vinculándose con problemas de transporte público y privado, conflictos entre usos del suelo destinados a la vivienda y a la actividad económica. El presente documento se ocupa de la transformación progresiva de la ciudad a través de los cambios en los usos del suelo. 
Estos cambios de uso pueden presentarse de manera diferenciada en el área urbana y, por lo tanto, se proponen modelos de probabilidad para analizar estas transformaciones.

De los resultados obtenidos se concluye que las variables espaciales, económicas y normativas son determinantes en los cambios de uso del suelo en la ciudad de Bogotá entre los años 2007 y 2017. Los resultados del modelo multinomial indican que la variable normativa que más incide en el cambio del uso $100 \%$ residencial al rango entre $99 \%$ y $80 \%$ es el tratamiento de consolidación con densificación moderada, ya que si una manzana es catalogada en el POT con este subtratamiento es $8,87 \%$ más probable que disminuya levemente la predominancia residencial. Mientras que la variable espacial que tiene mayor influencia en el cambio de uso del suelo son los Subcentros de empleo, debido a que un 1\% más cerca a los subcentros de empleo aumenta en 0,068\% la probabilidad de cambio.

La disminución en la predominancia de uso residencial implica que metros cuadrados construidos de las manzanas incorporadas en el modelo se destinaron en otro uso para 2017. Siete millones y medio de metros cuadrados de la categoría 1 y diez millones y medio de la categoría 2 se destinaron para otro tipo de usos, en la Figura 13 se observa la proporción de metros cuadrados por categoría que cambiaron a los otros usos como transporte-almacenamiento y comercio pequeño.

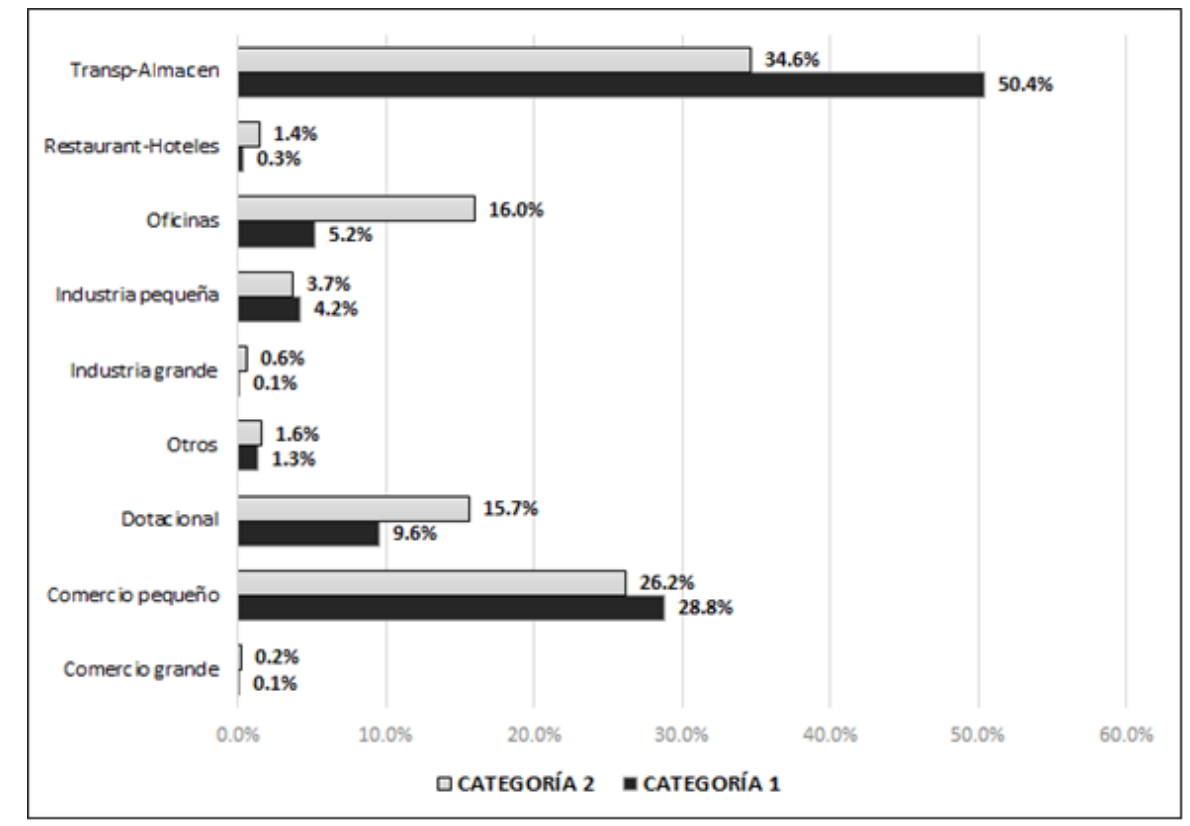

Figura 13 - Proporción de metros cuadrados que cambiaron. Fuente: Cálculos propios.

La matriz de transición muestra que en la ciudad está ocurriendo un cambio generalizado de manzanas con predominancia residencial a manzanas de uso mixto, lo que quiere decir que la disposición a pagar por el uso residencial en algunos puntos de la ciudad está igualando la disposición a pagar por otros usos y además existen incentivos para que usos como comercio pequeño invadan lugares con predominancia residencial.

Próximas investigaciones se enfocarán en el papel de la norma urbana, especialmente el nuevo POT para Bogotá, además de identificar potenciales determinantes que expliquen la configuración espacial de la ciudad.

\section{Agradecimientos}

El manuscrito fue financiado por la Universidad Sergio Arboleda y la Secretaría de Distrital de Planificación de Bogotá mediante el Acuerdo 369 de 2018: Desarrollo de un simulador para modelar la ocupación de la ciudad de Bogotá y la región; Sistema General de Regalías. BIPN código 2016000100031. 


\section{Referencias}

Akaike, H (1974). A new look at the statistical model identification. IEEE Transactions on Automatic Control, 19(6), 716-723. https://doi.org/10.1109/TAC.1974.1100705

Alonso, W. (1964). Location and Land Use (Reprint 2013 ed.). Harvard University Press. https://doi.org/10.4159/harvard.9780674730854

Avendaño, A., Enriquez, H., \& Olarte, S. (2014). Estructura urbana y precios del suelo en Bogotá. Economía y Región, 8(2), 4980 .

Bowes, D., \& Ihlanfeldt, K. (2001). Identifying the impacts of rail transit stations on residential property values. Journal of Urban Economics. 2001, 50(1), 1-25. https://doi.org/10.1006/juec.2001.2214

Congreso de Colombia (1997). Ley 388 de 1997. Establecer las bases para el ordenamiento del territorio. Colombia: Diario Oficial n. 43.127 .

Alcaldía Mayor de Bogotá (2004). Decreto 190 de 2004. Plan de Ordenamiento Territorial de Bogotá. Bogotá: Registro Distrital 3122.

Departamento administrativo de planeación (2016). Venta de derechos de construcción y desarrollo: Análisis de factibilidad. Alcaldía de Medellín.

Domínguez, A. (2017). Determinantes del precio y del cambio de uso del suelo. En Secretaría Distrital de Planeación, Análisis de la Dinámica Urbana para la planeación y el ordenamiento territorial. Alcaldía Mayor de Bogotá D.C.

Eliasson, J., Kopsch, F., Mandell, S., \& Wilhelmsson, M. (2020). Transport mode and the value of accessibility - A potential input for sustainable investment analysis. Sustainability, 12(5), 2143. https://doi.org/10.3390/su12052143

Fujita, M., \& Ogawa, H. (1982). Multiple equilibria and structural transition of non-monocentric urban configurations. Regional science and urban economics, 12(2), 161-196. https://doi.org/10.1016/0166-0462(82)90031-X

Fujita, M., \& Krugman, P. (2004). The new economic geography: Past, present and the future. En R. J. G. M. Florax, D. A. Plane (eds.). Fifty Years of Regional Science. Advances in Spatial Science (pp. 139-164). Springer. https://doi.org/10.1007/978-3-66207223-3_6.

Hausman, J., \& McFadden, D. (1984). Specification Tests for the Multinomial Logit Model. Econometrica, 52(5), 1219-1240. https://doi.org/10.2307/1910997

Jaramillo, S. (2009). Hacia una teoría de la renta del suelo urbano. Bogotá, Colombia: Editorial Universidad de los Andes, Bogotá - Colombia.

Koster, H. \& Rouwendal, J. (2013). Agglomeration, commuting costs, and the internal structure of cities. Regional science and urban economics, 43, 352-366. https://doi.org/10.1016/j.regsciurbeco.2012.09.001.

Kristof, F. (1970). Cities and Housing: The Spatial Pattern of Urban Residential Land Use. Chicago and London: The University of Chicago Press. Recuperado de 11 mayo, 2021 por http://www.jstor.org/stable/2720512

Krugman, P. (1991). Increasing Returns and Economic Geography. Journal of Political Economy, 99(31), 483-499. https://doi.org/10.1086/261763.

Lucas, R., \& Rossi-Hansberg, E. (2002). On the internal structure of cities. Econometrica, 70(4), 1445-1476. Recuperado 11 de mayo, 2021 por https://www.jstor.org/stable/3082004

McDonald, J., \& Prather, P. (1994). Suburban employment center-the case of Chicago. Urban studies, 31(2), $201-218$. Recuperado 11 mayo, 2021 por http://www.jstor.org/stable/43196088

McFadden, D. (1974). Conditional Logit Analysis of Qualitative Choice Behavior. En Zarembka (Ed.), Frontiers in econometrics (pp. 105-142). Nueva York: Academic Press.

McFadden, D. (1977). Modelling the choice of residential location. En A. Karlqvist, et al. (1978), Spatial interaction theory and planning models, Amsterdam, North Holland (pp. 75-96): Elsevier. 
Estructura urbana y cambio en el uso del suelo para Bogotá entre 2007 y 2017

McMillen, D. P. (2004). Employment densities, spatial autocorrelation, and subcenters in large metropolitan areas. Journal of regional science, 44, 225-244. https://doi.org/10.1111/j.0022-4146.2004.00335.x

Mills, E. S. (1967). An Aggregative Model of Resource Allocation in a Metropolitan Area. The American Economic Review, 57(2), 197-210. Recuperado 11 de mayo, 2021 por http://www.jstor.org/stable/1821621.

Muñiz, I., \& García-López, M. A. (2010). The polycentric knowledge economy in Barcelona. Urban Geography, 31(6), 774-799. https://doi.org/10.2747/0272-3638.31.6.774

Muñiz, I., García-López, M. A., \& Galindo, A. (2008). The effect of employment sub-centres on population density in Barcelona. Urban Studies, 45, 627-649. https://doi.org/10.1177/0042098007087338

Odland, J. (1978). The conditions for multi-center cities. Economic Geography, 54, 234-244. https://doi.org/10.2307/142837

Peréz, S., \& Polése, M. (1996). Modelos de análisis de planificación urbana: Estudios sobre la evolución y tendencias de la ciudad de puebla (1 $1^{\mathrm{a}}$ ed., Vol. 1). Plaza y Valdez editores.

Rodriguez, D. A., Vergel-Tovar, E., \& Camargo, W. F. (2016). Land development impacts of BRT in a sample of stops in Quito and Bogotá. Transport Policy, 51, 4-14. https://doi.org/10.1016/j.tranpol.2015.10.002

Schwarz, G. (1978). Estimating the dimension of a model. Annals of Statistics, 6, 461-464.

https://doi.org/10.1214/aos/1176344136

SDP (2021). Plan de Ordenamiento Territorial: Documento Resumen. Bogotá D.C.: Secretaría Distrital de Planeación -SDP. Recuperado el 11 de mayo de 2021, de http://www.sdp.gov.co

Vergel-Tovar (2016). Examining the reciprocal relationship between Bus Rapid Transit and the built environment in Latin America (Disertación de doctorado). Department of City and Regional Planning, University of North Carolina at Chapel Hill, Estados Unidos.

Von Thünen (1826). Von Thünen's Isolated State. Oxford: Pergamon Press.

Xu, Y., McNamara, P., Wu, Y., \& Dong, Y. (2013). An econometric analysis of changes in arable land utilization using multinomial logit model in Pinggu district Beijing. China. Journal of Environmental Management, 128, 324-334.

https://doi.org/10.1016/j.jenvman.2013.05.020

Zheng, S., Hu, X., Wang, J., \& Wang, R. (2016). Subways near the subway: Rail transit and neighborhood catering businesses in Beijing. Transport Policy, 2016(51), 81-92. https://doi.org/10.1016/j.tranpol.2016.03.008

Editor: Paulo Nascimento Neto

Recibido: 24 mayo 2020

Aprobado: 04 abr. 2021 


\section{Anexos}

Tabla A1 - Agrupación de tipos de usos del suelo

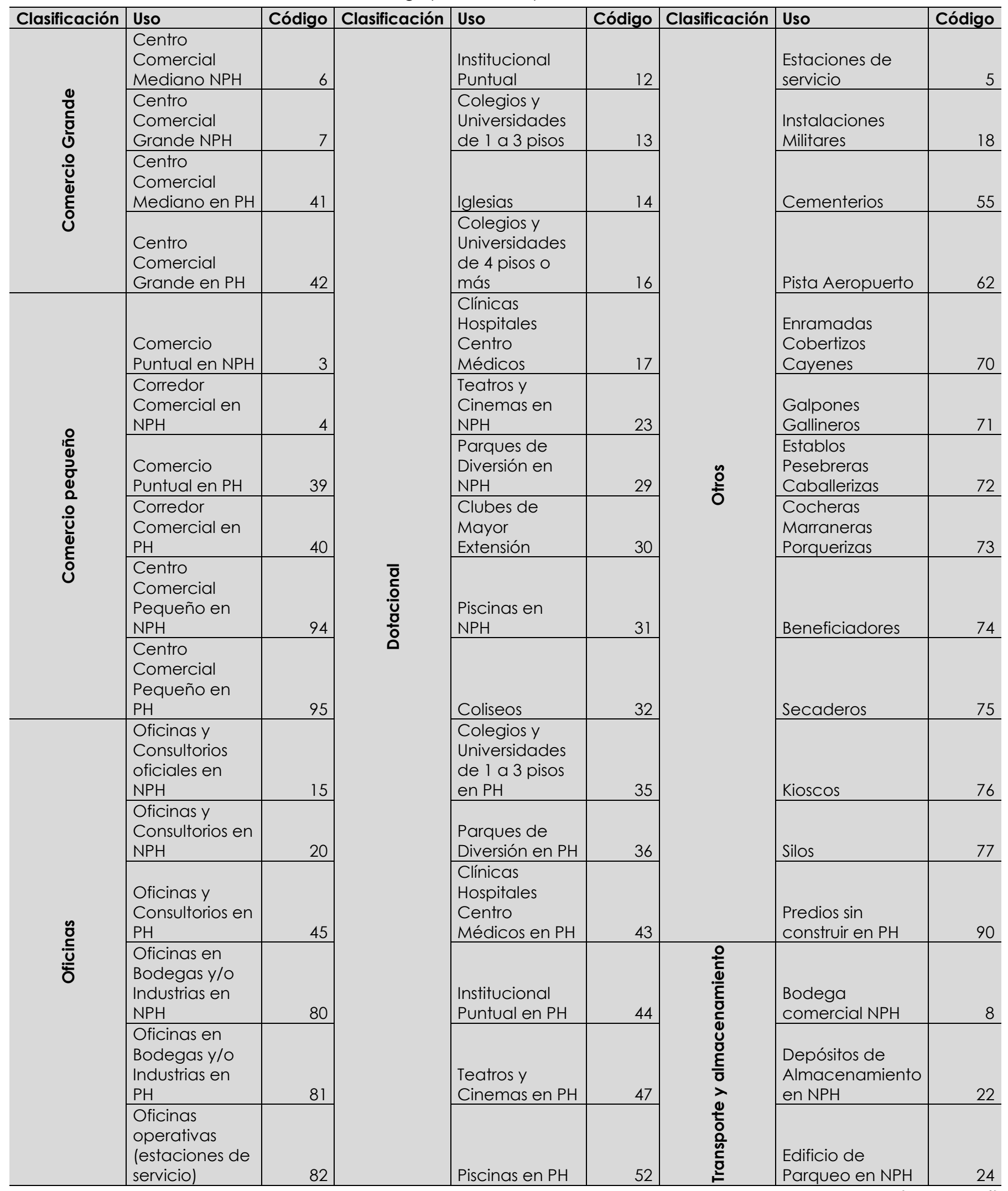


Tabla A1 - Agrupación de tipos de usos del suelo

(conclusión)

\begin{tabular}{|c|c|c|c|c|c|c|c|c|}
\hline Clasificación & Uso & Código & Clasificación & Uso & Código & Clasificación & Uso & Código \\
\hline Oficinas & \begin{tabular}{|l|} 
Oficinas y \\
Consultorios \\
oficiales en PH \\
\end{tabular} & 92 & \multirow{6}{*}{$\begin{array}{l}\bar{\delta} \\
\frac{0}{U} \\
\frac{0}{0} \\
0\end{array}$} & Iglesias en PH & 53 & \multirow{12}{*}{ 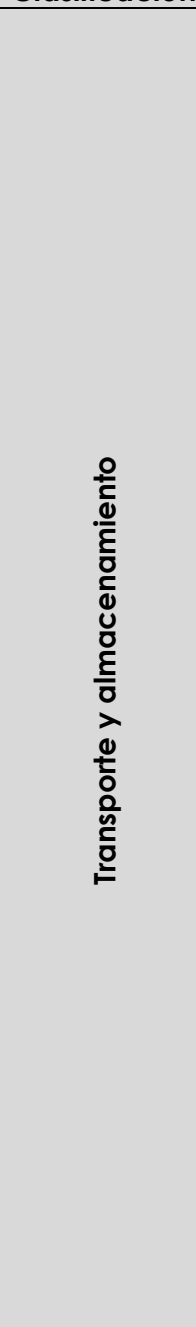 } & $\begin{array}{l}\text { Bodega de } \\
\text { Almacenamiento } \\
\text { en NPH }\end{array}$ & 25 \\
\hline \multirow{4}{*}{ 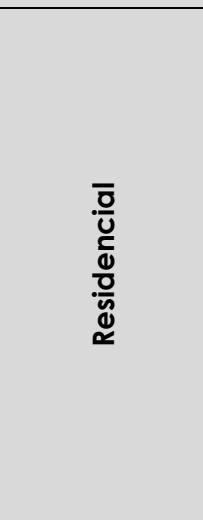 } & $\begin{array}{l}\text { Habitacional } \\
\text { menor o igual } \\
\text { a } 3 \text { pisos en } \\
\text { NPH } \\
\end{array}$ & 1 & & $\begin{array}{l}\text { Culto Religioso } \\
\text { en NPH }\end{array}$ & 58 & & $\begin{array}{l}\text { Bodega } \\
\text { Económica }\end{array}$ & 33 \\
\hline & $\begin{array}{l}\text { Habitacional } \\
\text { mayor o igual } \\
\text { a } 4 \text { pisos en } \\
\text { NPH } \\
\end{array}$ & 2 & & $\begin{array}{l}\text { Aulas de } \\
\text { Clases }\end{array}$ & 64 & & $\begin{array}{l}\text { Parqueo libre en } \\
\text { PH }\end{array}$ & 48 \\
\hline & \begin{tabular}{|l|} 
Habitacional \\
menor o igual \\
a 3 pisos en $\mathrm{PH}$ \\
\end{tabular} & 37 & & $\begin{array}{l}\text { Clubes } \\
\text { Pequeños }\end{array}$ & 65 & & $\begin{array}{l}\text { Parqueadero } \\
\text { Cubierto en PH }\end{array}$ & 49 \\
\hline & $\begin{array}{l}\text { Habitacional } \\
\text { mayor o igual } \\
\text { a } 4 \text { pisos en PH }\end{array}$ & 38 & & $\begin{array}{l}\text { Plazas de } \\
\text { Mercado }\end{array}$ & 66 & & $\begin{array}{l}\text { Edificio de } \\
\text { Parqueo en PH }\end{array}$ & 50 \\
\hline \multirow{4}{*}{ 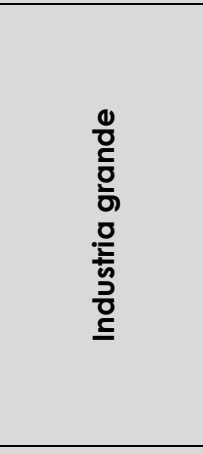 } & $\begin{array}{l}\text { Industria } \\
\text { Mediana }\end{array}$ & 10 & & Museos & 67 & & $\begin{array}{l}\text { Deposito Lockers } \\
\text { en PH }\end{array}$ & 51 \\
\hline & $\begin{array}{l}\text { Industria } \\
\text { Grande }\end{array}$ & 11 & \multirow{6}{*}{ 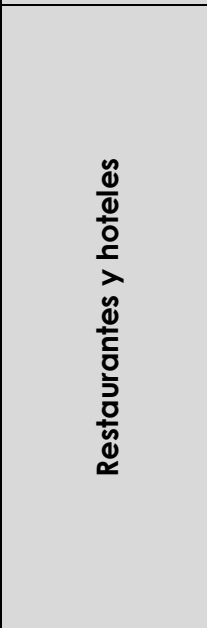 } & Hoteles en NPH & 21 & & $\begin{array}{l}\text { Bodega } \\
\text { comercial en PH }\end{array}$ & 91 \\
\hline & $\begin{array}{l}\text { Industria } \\
\text { Mediana en } \\
\text { PH }\end{array}$ & 28 & & \begin{tabular}{|l} 
Moteles \\
Amoblado y \\
Residencias en \\
NPH \\
\end{tabular} & 26 & & $\begin{array}{l}\text { Bodega de } \\
\text { Almacenamiento } \\
\text { en PH }\end{array}$ & 93 \\
\hline & $\begin{array}{l}\text { Industria } \\
\text { Grande en PH }\end{array}$ & 34 & & \begin{tabular}{|l|} 
Moteles \\
Amoblado y \\
Residencias en \\
PH
\end{tabular} & 27 & & $\begin{array}{l}\text { Parqueadero } \\
\text { Cubierto en NPH }\end{array}$ & 96 \\
\hline \multirow{3}{*}{ 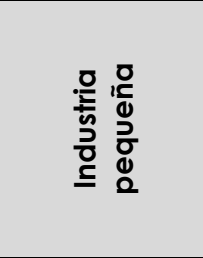 } & $\begin{array}{l}\text { Industria } \\
\text { artesanal } \\
\end{array}$ & 9 & & Hoteles en PH & 46 & & $\begin{array}{l}\text { Bodega } \\
\text { Económica en } \\
\text { PH }\end{array}$ & 97 \\
\hline & Industria & & & \begin{tabular}{|l|} 
Restaurantes \\
en NPH \\
\end{tabular} & 56 & & \multirow{2}{*}{$\begin{array}{l}\text { Depósito de } \\
\text { Almacenamiento } \\
\text { en PH }\end{array}$} & \multirow[b]{2}{*}{98} \\
\hline & $\begin{array}{l}\text { artesanal en } \\
\text { PH }\end{array}$ & 19 & & \begin{tabular}{|l} 
Restaurantes \\
en PH
\end{tabular} & 60 & & & \\
\hline
\end{tabular}

Fuente: Elaboración propia con base en la clasificación de IDECA (www.ideca.gov.co).

Tabla A2 - Criterios de información

\begin{tabular}{lll}
\hline variable & Mlogit & Mprobit \\
\hline Observaciones & 19910 & 19910 \\
Criterio de Akaike (AIC) & 29666,17 & 29824,14 \\
Criterio de Schwarz (BIC) & 29680,06 & 29838,04 \\
\hline
\end{tabular}

Fuente: Cálculos propios. 
Estructura urbana y cambio en el uso del suelo para Bogotá entre 2007 y 2017

Tabla A3 - Matriz de correlaciones

\begin{tabular}{lllllllllll}
\hline & Indtm & Indcc & Indmvi & Indsc & Curb & Cdensm & actcys & actres & point $x$ & point $y$ \\
\hline Indtm & 1 & & & & & & & & & \\
Indcc & 0.369 & 1 & & & & & & & & \\
Indmvi & 0.471 & 0.388 & 1 & & & & & & & \\
Indsc & 0.468 & 0.457 & 0.428 & 1 & & & & & \\
CUrb & -0.118 & -0.112 & -0.063 & -0.064 & 1 & & & & \\
Cdensm & -0.211 & -0.170 & -0.228 & -0.331 & -0.219 & 1 & & & \\
actcys & -0.277 & -0.239 & -0.221 & -0.294 & -0.099 & -0.022 & 1 & & \\
actres & 0.098 & 0.115 & 0.099 & 0.211 & 0.178 & 0.154 & -0.546 & 1 & & \\
point x & -0.207 & -0.219 & -0.335 & -0.546 & 0.082 & 0.123 & 0.176 & -0.089 & 1 \\
point y & -0.174 & -0.392 & -0.103 & -0.445 & 0.155 & 0.124 & 0.079 & -0.055 & 0.330 & 1 \\
\hline
\end{tabular}

Fuente: Cálculos propios. 